\title{
Nonlocal Theory of Ball Lightning
}

\author{
B. V. Alexeev \\ Moscow Technological University \\ Email: Boris.Vlad.Alexeev@gmail.com
}

\begin{abstract}
The existence in a bounded region of a self-consistent plasma object is established by the methods of non-local physics as a result of the solution of the Cauchy problem. The non-local theory is created for mathematical modeling of plasmoids and ball lightnings. The solitons have the character of the stable quantum objects in the self consistent electric field. Particularly these effects can be considered as explanation of the existence of lightning balls. The delivered theory demonstrates the great possibilities of the generalized quantum hydrodynamics in investigation of the quantum solitons. The theory leads to solitons as typical formations in the generalized quantum hydrodynamics. It is proved that all ball lightnings theories based on local description are wrong in principle.
\end{abstract}

Keywords: Foundations of the theory of transport processes, nonlocal quantum hydrodynamic equations, the theory of plasmoids, foundations of non-local physics, the theory of lighting balls.

\section{Introduction: about the Basic Principles of the Nonlocal Quantum Hydrodynamics}

The theory of transport processes (including quantum mechanics) can be considered in the frame of unified theory based on the non-local physical description (see, for example [1 - 5]). In particular the generalized hydrodynamic equations represent an effective tool for solving problems in the very vast area of physical problems. For simplicity in introduction, we will consider fundamental methodic aspects from the qualitative standpoint of view avoiding excessively cumbersome formulas. A rigorous description is found, for example, in the monograph [3]. Transport processes in open dissipative systems are considered in physical kinetics. Therefore, the kinetic description is inevitably related to the system diagnostics. Such an element of diagnostics in the case of theoretical description in physical kinetics is the concept of the physically infinitely small volume (PhSV). The correlation between theoretical description and system diagnostics is well-known in physics. Suffice it to recall the part played by test charge in electrostatics or by test circuit in the physics of magnetic phenomena. The traditional definition of PhSV contains the statement to the effect that the PhSV contains a sufficient number of particles for introducing a statistical description; however, at the same time, the $\mathbf{P h S V}$ is much smaller than the volume $V$ of the physical system under consideration; in a first approximation, this leads to local approach in investigating the transport processes. It is assumed in classical hydrodynamics that local thermodynamic equilibrium is first established within the PhSV, and only after that the transition occurs to global thermodynamic equilibrium if it is at all possible for the system under study. Let us consider the hydrodynamic description in more details from this point of view. Assume that we have two neighboring physically infinitely small volumes $\mathbf{P h S V}_{1}$ and $\mathbf{P h S V}_{2}$ in a non-equilibrium system.

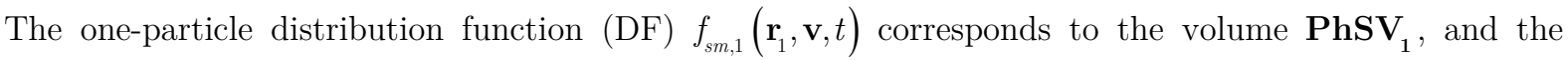
function $f_{s m, 2}\left(\mathbf{r}_{2}, \mathbf{v}, t\right)$ - to the volume $\mathbf{P h S V}_{2}$. It is assumed in a first approximation that $f_{s m, 1}\left(\mathbf{r}_{1}, \mathbf{v}, t\right)$ does not vary within $\mathbf{P h S V}_{\mathbf{1}}$, same as $f_{s m, 2}\left(\mathbf{r}_{2}, \mathbf{v}, t\right)$ does not vary within the neighboring volume $\mathrm{PhSV}_{2}$. It is this assumption of locality that is implicitly contained in the Boltzmann equation (BE). However, the assumption is too crude. Indeed, a particle on the boundary between two volumes, which experienced the last collision in $\mathbf{P h S V}_{\mathbf{1}}$ and moves toward $\mathbf{P h S V} \mathbf{V}_{2}$, introduces information about the $f_{s m, 1}\left(\mathbf{r}_{1}, \mathbf{v}, t\right)$ into the neighboring volume $\mathbf{P h S V}_{2}$. Similarly, a particle on the boundary between two 
volumes, which experienced the last collision in $\mathbf{P h S V}_{2}$ and moves toward $\mathbf{P h S V}$, introduces information about the DF $f_{s m, 2}\left(\mathbf{r}_{2}, \mathbf{v}, t\right)$ into the neighboring volume $\mathbf{P h S V}_{1}$. The relaxation over translational degrees of freedom of particles of like masses occurs during several collisions. As a result, "Knudsen layers" are formed on the boundary between neighboring physically infinitely small volumes, the characteristic dimension of which is of the order of path length. Therefore, a correction must be introduced into the DF in the $\mathbf{P h S V}$, which is proportional to the mean time between collisions and to the substantive derivative of the DF being measured (rigorous derivation is given in [3]). Let a particle of finite radius be characterized as before by the position $\mathbf{r}$ at the instant of time $t$ of its center of mass moving at velocity $\mathbf{v}$. Then, the situation is possible where, at some instant of time $t$, the particle is located on the interface between two volumes. In so doing, the lead effect is possible (say, for $\mathbf{P h S V}_{2}$ ), when the center of mass of particle moving to the neighboring volume $\mathbf{P h S V}_{2}$ is still in $\mathbf{P h S V}_{1}$. However, the delay effect takes place as well, when the center of mass of particle moving to the neighboring volume (say, $\mathbf{P h S V}_{2}$ ) is already located in $\mathbf{P h S V}_{2}$ but a part of the particle still belongs to $\mathbf{P h S V}_{1}$. This entire complex of effects defines non-local effects in space and time. The physically infinitely small volume (PhSV) is an open thermodynamic system for any division of macroscopic system by a set of PhSVs. However, the BE [1-3]

$$
D f / D t=J^{B}
$$

where $J^{B}$ is the Boltzmann collision integral and $D / D t$ is a substantive derivative, fully ignores nonlocal effects and contains only the local collision integral $J^{B}$. The foregoing nonlocal effects are insignificant only in equilibrium systems, where the kinetic approach changes to methods of statistical mechanics.

This is what the difficulties of classical Boltzmann physical kinetics arise from. Also a weak point of the classical Boltzmann kinetic theory is the treatment of the dynamic properties of interacting particles. On the one hand, as follows from the so-called "physical" derivation of the BE, Boltzmann particles are regarded as material points; on the other hand, the collision integral in the BE leads to the emergence of collision cross sections.

Results:

1) the kinetic theory should be non-local theory;

2) the effect is of the order of the Knudsen number;

3) the effect is due to the reduced description and is not related to the specific division of the physical system by grid $\mathrm{PhSV}$;

4) accurate derivation of the kinetic equation with respect to a one-particle distribution function (DF) should lead to corrections of the order of the Knudsen number before the Bogolyubov chain is uncoupled; 5) this means that in the Boltzmann equation the terms of the Knudsen number order are lost, which are essential for both large and small Knudsen numbers;

6) the Boltzmann equation does not belong even to the class of minimal models, being only a "plausible" equation;

7) the Boltzmann equation in this sense is the wrong equation.

It is clear that this is a revolution in the theory of dissipative processes, in particular in hydrodynamics $[1-3]$. In the hydrodynamic Navier-Stokes equation, which is a direct consequence of the Boltzmann equation, one-order (by Knudsen number) terms are lost, which in the classical NavierStokes equation contain viscosity. As a result we have problems with the turbulence at all and problems of existence and uniqueness of solutions of the Navier-Stokes equations, in particular.

Abstract of the classical Madelung article [6] contains only one brilliant phrase: "It is shown that the Schrödinger equation (SE) for one-electron problems can be transformed into the form of hydrodynamic equations". However, practically the hydrodynamics of Madelung was forgotten. There were (incorrect) opinions that quantization cannot be introduced into the fluid dynamics of Madelung. It is interesting to note that in the famous book of David Bohm [7] ("Quantum Theory", New York, Prentice-Hall, Inc., 1952), containing 728 pages in the Russian translation, the name of Madelung is not even mentioned!

Is it possible to obtain SE from Liouville kinetic equation? The answer to this question is negative. It is established that the Liouville equation can be transformed into SE only after the artificial introduction of spatial nonlocality without the introduction of nonlocality in time. 
The following conclusions of fundamental importance can be made:

1. The quantum hydrodynamics of Madelung is equivalent to the Schrödinger equation and leads to a description of the evolution of the quantum particle in the form of the motion equation and the continuity equation. SE is a consequence of the nonlocal Liouville equation as a result of the artificial introduction of the local spatial approximation of the nonlocal equations.

2. The Fundamental Nonlocal Equation (FNE, Alexeev equation [1 - 4]) contains strict accounting of nonlocal effects in space and time and after the limit transition to the local approximation leads to the non-locality parameter $\tau$, which at the quantum level is consistent with the Heisenberg uncertainty principle.

The disadvantages of the Schrödinger equation:

1. The Schrödinger equation is a postulate. Another differentiation of the function leads to other equations, for example, to equations containing the second derivative of time.

2. The Schrödinger equation does not describe dissipative processes.

3. The Schrödinger equation is not able to describe the whole complex "nucleus-electron shell".

4. The Schrödinger equation is unable to describe a spatial electron shell without using of additional assumptions such as the Pauli principle.

5. To a large extent, the quantization result is the result of cutting infinite series and turning them into polynomials. This process resembles the transformation of a traveling wave into a standing wave system, if you enter a reflection from an obstacle.

Generalized hydrodynamic equations (GHE) should contain Schrödinger equation (SE) as a deep special case. This affirmation was proved in [2 - 4]. In other words, we formulated in explicit form all assumptions (all steps) that should be implemented to obtain SE from the FNE and GHE. At the final stage, for simplicity, a non - stationary 1D model of the Madelung-Schrödinger was obtained without taking into account external forces.

So, we can state that SE is a deep special case of generalized hydrodynamic equations. This means that a new quantum mechanics of dissipative processes has been created.

Note again that the Schrödinger equation and its equivalent hydrodynamic form of Madelung do not describe dissipative processes and therefore cannot be effectively used in nanotechnology. The Boltzmann equation essentially "does not work" at the distances of the order of the radius of interaction of particles and, therefore, can not be effectively used in the theoretical study of nanotechnology even in the framework of "plausible" models.

FNE have extremely important for astrophysics special cases when density $\rho \rightarrow 0$ (the initial stage of evolution of the Universe, the Big Bang) and when density $\rho \rightarrow \infty$ (evolution of the black hole). Both limiting cases have no physical or mathematical meaning in "classical" hydrodynamics. Thus, we have a unified statistical theory of dissipative structures, which has a hydrodynamic shape.

The rigorous approach to derivation of kinetic equation relative to one-particle $\operatorname{DF} f\left(K E_{f}\right)$ is based on employing the hierarchy of Bogoliubov equations. Generally speaking, the structure of $K E_{f}$ is as follows:

$$
\frac{D f}{D t}=J^{B}+J^{n l}
$$

where $J^{n l}$ is the non-local integral term. An approximation for the second collision integral is suggested by me in generalized Boltzmann physical kinetics,

$$
J^{n l}=\frac{D}{D t}\left(\tau \frac{D f}{D t}\right)
$$

Here, $\tau$ is nonlocal parameter, which in the simplest case is equal to the mean time between collisions of particles, which is related in a hydrodynamic approximation with dynamical viscosity $\mu$ and pressure $p$,

$$
\tau p=\Pi \mu
$$

where the factor $\Pi$ is defined by the model of collision of particles: for neutral hard-sphere gas, $\Pi=0.8$ [8]. All of the known methods of deriving kinetic equation relative to one-particle DF lead to approximation (1.3), including the method of many scales, the method of correlation functions, and the iteration method. Fluctuation effects occur in any open thermodynamic system bounded by a control 
surface transparent to particles. Generalized BE (1.2) leads to generalized hydrodynamic equations (GHE) [1-3] as the local approximation of non local effects, for example, to the continuity equation

$$
\frac{\partial \rho^{a}}{\partial t}+\frac{\partial}{\partial \mathbf{r}} \cdot\left(\rho \mathbf{v}_{0}\right)^{a}=0
$$

where $\rho^{a}, \mathbf{v}_{0}{ }^{a},\left(\rho \mathbf{v}_{0}\right)^{a}$ "average values" and should be calculated in view of non-locality effect in terms of gas density $\rho$, hydrodynamic velocity of flow $\mathbf{v}_{0}$, and density of momentum flux $\mathbf{v}_{0}$; for locally Maxwellian distribution, $\rho^{a},\left(\rho \mathbf{v}_{0}\right)^{a}$ are defined by the relations

$$
\left(\rho-\rho^{a}\right) / \tau=\frac{\partial \rho}{\partial t}+\frac{\partial}{\partial \mathbf{r}} \cdot\left(\rho \mathbf{v}_{0}\right),\left(\rho \mathbf{v}_{0}-\left(\rho \mathbf{v}_{0}\right)^{a}\right) / \tau=\frac{\partial}{\partial t}\left(\rho \mathbf{v}_{0}\right)+\frac{\partial}{\partial \mathbf{r}} \cdot \rho \mathbf{v}_{0} \mathbf{v}_{0}+\overrightarrow{\mathrm{I}} \cdot \frac{\partial p}{\partial \mathbf{r}}-\rho \mathbf{a}
$$

where $\overrightarrow{\mathrm{I}}$ is a unit tensor, and a is the acceleration due to the effect of mass forces. In the general case, the parameter $\tau$ is the non-locality parameter; in hydrodynamics it corresponds to the relaxation time [1]. The violation of Bell's inequalities [9] is found for local statistical theories, and the transition to nonlocal description is inevitable. The following conclusion of principal significance can be done from the previous consideration [1-4]:

1.Madelung's quantum hydrodynamics is equivalent to the Schrödinger equation (SE) and furnishes the description of the quantum particle evolution in the form of Euler equation and continuity equation.

2.Generalized Boltzmann physical kinetics brings the strict approximation of non-local effects in space and time and after transmission to the local approximation leads to parameter $\tau$, which on the quantum level corresponds to the uncertainty principle "time-energy".

3.GHE produce SE as a deep particular case of the generalized Boltzmann physical kinetics and therefore of non-local hydrodynamics.

In principal no needs in using of the "time-energy" uncertainty relation for estimation of the value of the non-locality parameter $\tau$ for GHE. Moreover the "time-energy" uncertainty relation does not produce the exact relations and from position of non-local physics is only the simplest estimation of the non-local effects. Really, let us consider two neighboring physically infinitely small volumes $\mathbf{P h S V}_{1}$ and $\mathrm{PhSV}_{2}$ in a non-equilibrium system. Obviously the time $\tau$ should tend to diminish with increasing of the velocities $u$ of particles invading in the nearest neighboring physically infinitely small volume $\left(\mathrm{PhSV}_{1}\right.$ or $\left.\mathrm{PhSV}_{2}\right)$ :

$$
\tau=H / u^{n}
$$

But the value $\tau$ cannot depend on the velocity direction and naturally to tie $\tau$ with the particle kinetic energy, then

$$
\tau=H / m u^{2}
$$

where $H$ is a coefficient of proportionality, which reflects the state of physical system. In the simplest case $H$ is equal to Plank constant $\hbar$ and relation (1.8) became compatible with the Heisenberg relation.

Important to notice that approximation (1.8) is compatible not only with the Heisenberg relation but also with the theory of the differencing scheme stability. Under the differencing scheme is usually understood a system of differential equations, (with additional conditions) chosen for description a particular problem of mathematical physics. Let some linear non-stationary problem of mathematical physics be considered. It can be interpreted as an abstract Cauchy problem

$$
B(t) \frac{d u(t)}{d t}+A(t) u(t)=f(t), 0<t \leq T, u(0)=u_{0} ;
$$

here operators $A(t)$ and $B(t)$ defined on everywhere dense set of Banach spaces $\mathrm{S}_{\mathrm{B}}$. When the initial problem is approximated by a finite difference scheme, the space $S_{B}$ is replaced by a family of finitedimensional spaces $S_{\mathrm{f}}$. The segment $[0, T]$ is replaced by the grid

$$
t_{n}=n \tau ; n=0,1, \ldots K ; K \tau=T .
$$


The operators $A(t)$ and $B(t)$ are replaced by difference operators acting in space $\mathrm{S}_{\mathrm{f}}$, and derivative $\frac{d u(t)}{d t}$ - by a difference relation $\frac{u\left(t_{n+1}\right)-u\left(t_{n}\right)}{\tau}$. As a result, we obtain the operator-difference equation

$$
B y_{t}+A y=\phi, \quad y(t=0)=y_{0}
$$

Equation (1.10) is a two-layer finite-difference scheme, because it binds the values of the unknown value $y(t)$ on two time layers $t=t_{n}$ and $t=t_{n+1}$. The record of a two-layer difference scheme in the form of (3.2) is known as a canonical form. Along with (1.10) we consider the equation with zero the right-hand side

$$
B y_{t}+A y=0, \quad y(t=0)=y_{0}
$$

A two-layer difference scheme (1.11) is defined in the linear space $S_{f}$. Suppose that in $S_{f}$ the norm $\|y(t)\|$ is introduced, which measures the solution of problem (1.11).

Definition 1. Scheme (1.11) is called stable if there exists constant M, independent of the numerical steps in space and time such that for any $y_{0}$ the solution of the problem (1.1) can be estimated as

$$
\left\|y\left(t_{n+1}\right)\right\| \leq \mathrm{M}\|y(0)\|
$$

It means that in this case scheme (1.12) is stable on initial data. Let in Gibert space $\mathrm{H}$ be given a selfadjoint positive operator D.

Definition 2. The energy space $\mathrm{H}_{\mathrm{D}}$ is the linear space $\mathrm{H}$ in which the scalar product $(y, v)_{\mathrm{D}}=(\mathrm{D} y, v)$ and the norm $\|y\|_{\mathrm{D}}=\sqrt{(\mathrm{D} y, y)}$ are introduced.

Definition 3. The difference scheme (1.11) is called a stable in $H_{D}$ space, if any $y_{n} \in H_{D}$ and all $n=0,1, \ldots$ for the solution of $y_{n+1}$ of problem (1.11) the estimation

$$
\left\|y_{n+1}\right\|_{\mathrm{D}} \leq\left\|y_{n}\right\|_{\mathrm{D}}
$$

is valid.

Thus, stability in this sense is uniform stability according to initial data, and the constant $M$ is equal to one (see definition (3.4)). We consider scheme (1.11) with operators $A$ and $B$ acting in Hilbert space $\mathrm{H}$. We formulate a basic theorem that proves the stability of the scheme (3.3) in the energetic space $\mathrm{H}_{A}$ constructed by the operator $A$ of the scheme (1.1).

Theorem. (A. A. Samarskii, [10])

Let the operator $A$ is self-adjoint and positive operator and does not depend on $n$. If exists the operator $B^{-1}$ then for stability of the scheme (1.11) in $\mathrm{H}_{A}$ it is necessary and sufficient the implementation of operator non-equality

$$
\begin{gathered}
B_{0} \geq \frac{1}{2} \tau A \\
B_{0}=\frac{1}{2}\left(B+B^{*}\right)
\end{gathered}
$$

Interesting to notice that [10] contains two different proofs of this theorem based on the energetic inequalities and on estimations of the norm of the transition operator.

We give an interpretation of this inequality (3.6) from a physical point of view. The relation (1.15) can be written in other form supposing that $B$ is a real operator

$$
\frac{1}{2} \tau A \leq B
$$

Let $\frac{1}{2} A=E$ correspond to the energy of a physical system and $B=\hbar$ is Planck constant in the limit case. We reach the relation

$$
\tau_{s} E \leq \hbar
$$

where $\tau_{s}$ is a time step in the difference scheme. In particular 


$$
\frac{1}{2} \tau_{s} u^{2} \leq \frac{\hbar}{m_{e}}
$$

where

$$
v_{e, q u}=\frac{\hbar}{m_{e}}=1.1577 \frac{\mathrm{cm}^{2}}{\mathrm{~s}}
$$

is the quantum kinematic viscosity for electron component. Let us denote

$$
v_{\text {mesh }}=\frac{1}{2} \tau_{s} u^{2}
$$

as a mesh kinematic viscosity. Then in this case it should be

$$
v_{\text {mesh }} \leq v_{e, q u}
$$

The well-known Heisenberg's uncertainty principle can be written in the form "energy - time"

$$
\tau_{H} E \geq \hbar
$$

Let us denote also

$$
v_{H}=\frac{1}{2} \tau_{H} u^{2}
$$

Then the choice of the approximations in the form (1.8) is reasonable also from the position of the numerical calculations on the quantum level and for the electron species corresponds to relation

$$
v_{\text {mesh }}=v_{e, q u}=v_{e, H}
$$

More details in the theory of the $\tau$ approximation can be found in $[1-4]$. Approximations for a nonlocal parameters $\tau_{i}, \tau_{e}$ and $\tau_{e i}$, need an additional explanation. The following approximations for $\tau_{i}$ and $\tau_{e}$

$$
\tau_{i}=H / m_{i} u^{2}, \quad \tau_{e}=H / m_{e} u^{2}
$$

correspond to the (1.8). For non-local interaction parameter of positive and negative particles (in particular, the electron - ion interaction) $\tau_{e i}$ is applicable the ratio

$$
\frac{1}{\tau_{e i}}=\frac{1}{\tau_{e}}+\frac{1}{\tau_{i}}
$$

In this case, the parameter $\tau_{e i}$ corresponds to the relaxation time of different species in the process of interaction. Conversion (1.26) is consistent with the Heisenberg's uncertainty principle if $H=\hbar$. From (3.20) follows

Then

$$
\frac{1}{\tau_{e i}}=\frac{\tau_{e}+\tau_{i}}{\tau_{e} \tau_{i}}=\frac{\frac{\hbar}{m_{e} u^{2}}+\frac{\hbar}{m_{i} u^{2}}}{\frac{\hbar^{2}}{u^{4}} \frac{1}{m_{e} m_{i}}}=\frac{u^{2}}{\hbar}\left(m_{e}+m_{i}\right)
$$

$$
u^{2}\left(m_{e}+m_{i}\right) \tau_{e i}=\hbar
$$

Equation (1.28) is an obvious consequence of the uncertainty relation for the combined particle whose mass is equal to $m_{i}+m_{e}$. But some remarks of the principal significance should be done.

It is known that Ehrenfest adiabatic theorem is one of the most important and widely studied theorems in Schrödinger quantum mechanics. It states that if we have a slowly changing Hamiltonian that depends on time, and the system is prepared in one of the instantaneous eigenstates of the Hamiltonian then the state of the system at any time is given by an the instantaneous eigenfunction of the Hamiltonian up to multiplicative phase factors. It can be formulated by other words. Let us consider the dynamic system with the Hamilton function

$H(p, q ; \lambda)$, where $p=p_{1}, \ldots, p_{n} ; q=q_{1}, \ldots, q_{n}$ and $\lambda=v_{\tau} \tau$. 
Definition. Function $J(p, q ; \lambda)$ is adiabatic invariant for the considered system, if the value $J(t)=J[p(t), q(t) ; \lambda(t)]$ is a little changing value over time $\tau \sim \frac{1}{v_{\tau}}$.

The adiabatic theory can be naturally incorporated in generalized quantum hydrodynamics based on local approximations of non-local terms. In the simplest case if $\Delta Q$ is the elementary heat quantity delivered for a system executing the transfer from one state (the corresponding time moment is $t_{i n}$ ) to the next one (the time moment $t_{e}$ ) then

$$
\Delta Q=\frac{1}{\tau} 2 \delta(\bar{T} \tau)
$$

where $\tau=t_{e}-t_{i n}$ and $\bar{T}$ is the average kinetic energy. This approach was discussed firstly by L. Boltzmann [11]. Obviously, each exactly conserved (invariant) quantity is an adiabatic invariant.

For example L. Brillouin (Chapter 7 "Boltzmann's formula and adiabatic invariants" in [12]) writes "We have shown that for all systems that are capable of oscillating in accord with a purely-sinusoidal law, the quantity $\tau E$ will be an adiabatic invariant, where $E$ represents the total energy. It was to such systems that Planck first applied the idea of quanta by writing that

$$
\tau E=\frac{E}{v}=n h, n \quad \text { integer }
$$

For these pure oscillators, the total energy will then be equal to an integer number times $h v . "$

For adiabatic case Ehrenfest supposes that

$$
2 \bar{T} \tau=\Omega_{1}, \Omega_{2}, \ldots
$$

where $\Omega_{1}, \Omega_{2}, \ldots$ are adiabatic invariants. Obviously for Plank's oscillator

$$
2 \bar{T} \tau=n h
$$

Then the adiabatic theorem and consequences of this theory deliver the general quantization conditions for non-local quantum hydrodynamics, in particular

$$
\begin{gathered}
\tau_{e}=\frac{n}{v_{e}} \text { for electronic shell, } \\
\tau_{e}=\frac{k}{v_{i}} \text { for nucleus, }
\end{gathered}
$$

where $n$ is shell quantum number and $k$ is nucleus quantum number.

\section{Basic Equations}

Strict consideration leads to the following system of the nonlocal hydrodynamic equations (GHE) [1 - 4] written in the generalized Euler form:

continuity equation for species $\alpha$

$$
\begin{aligned}
& \frac{\partial}{\partial t}\left\{\rho_{\alpha}-\tau_{\alpha}\left[\frac{\partial \rho_{\alpha}}{\partial t}+\frac{\partial}{\partial \mathbf{r}} \cdot\left(\rho_{\alpha} \mathbf{v}_{0}\right)\right]\right\}+\frac{\partial}{\partial \mathbf{r}} \cdot\left\{\rho_{\alpha} \mathbf{v}_{0}-\tau_{\alpha}\left[\frac{\partial}{\partial t}\left(\rho_{\alpha} \mathbf{v}_{0}\right)+\frac{\partial}{\partial \mathbf{r}} \cdot\left(\rho_{\alpha} \mathbf{v}_{0} \mathbf{v}_{0}\right)+\overrightarrow{\mathrm{I}} \cdot \frac{\partial p_{\alpha}}{\partial \mathbf{r}}\right.\right. \\
& \left.\left.-\rho_{\alpha} \mathbf{F}_{\alpha}^{(1)}-\frac{q_{\alpha}}{m_{\alpha}} \rho_{\alpha} \mathbf{v}_{0} \times \mathbf{B}\right]\right\}=R_{\alpha},
\end{aligned}
$$

and continuity equation for mixture

Momentum equation for species

$$
\begin{aligned}
& \frac{\partial}{\partial t}\left\{\rho-\sum_{\alpha} \tau_{\alpha}\left[\frac{\partial \rho_{\alpha}}{\partial t}+\frac{\partial}{\partial \mathbf{r}} \cdot\left(\rho_{\alpha} \mathbf{v}_{0}\right)\right]\right\}+\frac{\partial}{\partial \mathbf{r}} \cdot\left\{\rho \mathbf{v}_{0}-\sum_{\alpha} \tau_{\alpha}\left[\frac{\partial}{\partial t}\left(\rho_{\alpha} \mathbf{v}_{0}\right)+\frac{\partial}{\partial \mathbf{r}} \cdot\left(\rho_{\alpha} \mathbf{v}_{0} \mathbf{v}_{0}\right)\right.\right. \\
& \left.\left.+\overrightarrow{\mathrm{I}} \cdot \frac{\partial p_{\alpha}}{\partial \mathbf{r}}-\rho_{\alpha} \mathbf{F}_{\alpha}^{(1)}-\frac{q_{\alpha}}{m_{\alpha}} \rho_{\alpha} \mathbf{v}_{0} \times \mathbf{B}\right]\right\}=0 .
\end{aligned}
$$




$$
\begin{aligned}
& \frac{\partial}{\partial t}\left\{\rho_{\alpha} \mathbf{v}_{0}-\tau_{\alpha}\left[\frac{\partial}{\partial t}\left(\rho_{\alpha} \mathbf{v}_{0}\right)+\frac{\partial}{\partial \mathbf{r}} \cdot \rho_{\alpha} \mathbf{v}_{0} \mathbf{v}_{0}+\frac{\partial p_{\alpha}}{\partial \mathbf{r}}-\rho_{\alpha} \mathbf{F}_{\alpha}^{(1)}-\frac{q_{\alpha}}{m_{\alpha}} \rho_{\alpha} \mathbf{v}_{0} \times \mathbf{B}\right]\right\} \\
& -\mathbf{F}_{\alpha}^{(1)}\left[\rho_{\alpha}-\tau_{\alpha}\left(\frac{\partial \rho_{\alpha}}{\partial t}+\frac{\partial}{\partial \mathbf{r}}\left(\rho_{\alpha} \mathbf{v}_{0}\right)\right)\right] \\
& -\frac{q_{\alpha}}{m_{\alpha}}\left\{\rho_{\alpha} \mathbf{v}_{0}-\tau_{\alpha}\left[\frac{\partial}{\partial t}\left(\rho_{\alpha} \mathbf{v}_{0}\right)+\frac{\partial}{\partial \mathbf{r}} \cdot \rho_{\alpha} \mathbf{v}_{0} \mathbf{v}_{0}+\frac{\partial p_{\alpha}}{\partial \mathbf{r}}-\rho_{\alpha} \mathbf{F}_{\alpha}^{(1)}-\frac{q_{\alpha}}{m_{\alpha}} \rho_{\alpha} \mathbf{v}_{0} \times \mathbf{B}\right]\right\} \times \mathbf{B} \\
& +\frac{\partial}{\partial \mathbf{r}} \cdot\left\{\rho_{\alpha} \mathbf{v}_{0} \mathbf{v}_{0}+p_{\alpha} \overrightarrow{\mathrm{I}}-\tau_{\alpha}\left[\frac{\partial}{\partial t}\left(\rho_{\alpha} \mathbf{v}_{0} \mathbf{v}_{0}+p_{\alpha} \overrightarrow{\mathrm{I}}\right)+\frac{\partial}{\partial \mathbf{r}} \cdot \rho_{\alpha}\left(\mathbf{v}_{0} \mathbf{v}_{0}\right) \mathbf{v}_{0}+2 \overrightarrow{\mathrm{I}}\left(\frac{\partial}{\partial \mathbf{r}} \cdot\left(p_{\alpha} \mathbf{v}_{0}\right)\right)\right.\right. \\
& \left.\left.+\frac{\partial}{\partial \mathbf{r}} \cdot\left(I \vec{I} p_{\alpha} \mathbf{v}_{0}\right)-\mathbf{F}_{\alpha}^{(1)} \rho_{\alpha} \mathbf{v}_{0}-\rho_{\alpha} \mathbf{v}_{0} \mathbf{F}_{\alpha}^{(1)}-\frac{q_{\alpha}}{m_{\alpha}} \rho_{\alpha}\left[\mathbf{v}_{0} \times \mathbf{B}\right] \mathbf{v}_{0}-\frac{q_{\alpha}}{m_{\alpha}} \rho_{\alpha} \mathbf{v}_{0}\left[\mathbf{v}_{0} \times \mathbf{B}\right]\right]\right\} \\
& =\int m_{\alpha} \mathbf{v}_{\alpha} J_{\alpha}^{s t, e l} d \mathbf{v}_{\alpha}+\int m_{\alpha} \mathbf{v}_{\alpha} J_{\alpha}^{s t, i n e l} d \mathbf{v}_{\alpha} .
\end{aligned}
$$

Generalized moment equation for mixture

$$
\begin{aligned}
& \frac{\partial}{\partial t}\left\{\rho \mathbf{v}_{0}-\sum_{\alpha} \tau_{\alpha}\left[\frac{\partial}{\partial t}\left(\rho_{\alpha} \mathbf{v}_{0}\right)+\frac{\partial}{\partial \mathbf{r}} \cdot \rho_{\alpha} \mathbf{v}_{0} \mathbf{v}_{0}+\frac{\partial p_{\alpha}}{\partial \mathbf{r}}-\rho_{\alpha} \mathbf{F}_{\alpha}^{(1)}-\frac{q_{\alpha}}{m_{\alpha}} \rho_{\alpha} \mathbf{v}_{0} \times \mathbf{B}\right]\right\} \\
& -\sum_{\alpha} \mathbf{F}_{\alpha}^{(1)}\left[\rho_{\alpha}-\tau_{\alpha}\left(\frac{\partial \rho_{\alpha}}{\partial t}+\frac{\partial}{\partial \mathbf{r}}\left(\rho_{\alpha} \mathbf{v}_{0}\right)\right)\right] \\
& -\sum_{\alpha} \frac{q_{\alpha}}{m_{\alpha}}\left\{\rho_{\alpha} \mathbf{v}_{0}-\tau_{\alpha}^{(0)}\left[\frac{\partial}{\partial t}\left(\rho_{\alpha} \mathbf{v}_{0}\right)+\frac{\partial}{\partial \mathbf{r}} \cdot \rho_{\alpha} \mathbf{v}_{0} \mathbf{v}_{0}+\frac{\partial p_{\alpha}}{\partial \mathbf{r}}-\rho_{\alpha} \mathbf{F}_{\alpha}^{(1)}-\frac{q_{\alpha}}{m_{\alpha}} \rho_{\alpha} \mathbf{v}_{0} \times \mathbf{B}\right]\right\} \times \mathbf{B} \\
& +\frac{\partial}{\partial \mathbf{r}} \cdot\left\{\rho \mathbf{v}_{0} \mathbf{v}_{0}+p \overrightarrow{\mathrm{I}}-\sum_{\alpha} \tau_{\alpha}\left[\frac{\partial}{\partial t}\left(\rho_{\alpha} \mathbf{v}_{0} \mathbf{v}_{0}+p_{\alpha} \overrightarrow{\mathrm{I}}\right)+\frac{\partial}{\partial \mathbf{r}} \cdot \rho_{\alpha}\left(\mathbf{v}_{0} \mathbf{v}_{0}\right) \mathbf{v}_{0}+2 \overrightarrow{\mathrm{I}}\left(\frac{\partial}{\partial \mathbf{r}} \cdot\left(p_{\alpha} \mathbf{v}_{0}\right)\right)+\frac{\partial}{\partial \mathbf{r}} \cdot\left(\tilde{\mathrm{I}} p_{\alpha} \mathbf{v}_{0}\right)\right.\right. \\
& \left.\left.-\mathbf{F}_{\alpha}^{(1)} \rho_{\alpha} \mathbf{v}_{0}-\rho_{\alpha} \mathbf{v}_{0} \mathbf{F}_{\alpha}^{(1)}-\frac{q_{\alpha}}{m_{\alpha}} \rho_{\alpha}\left[\mathbf{v}_{0} \times \mathbf{B}\right] \mathbf{v}_{0}-\frac{q_{\alpha}}{m_{\alpha}} \rho_{\alpha} \mathbf{v}_{0}\left[\mathbf{v}_{0} \times \mathbf{B}\right]\right]\right\}=0
\end{aligned}
$$

Energy equation for component

$$
\begin{aligned}
& \frac{\partial}{\partial t}\left\{\frac{\rho_{\alpha} v_{0}^{2}}{2}+\frac{3}{2} p_{\alpha}+\varepsilon_{\alpha} n_{\alpha}-\tau_{\alpha}\left[\frac{\partial}{\partial t}\left(\frac{\rho_{\alpha} v_{0}^{2}}{2}+\frac{3}{2} p_{\alpha}+\varepsilon_{\alpha} n_{\alpha}\right)\right.\right. \\
& \left.\left.+\frac{\partial}{\partial \mathbf{r}} \cdot\left(\frac{1}{2} \rho_{\alpha} v_{0}^{2} \mathbf{v}_{0}+\frac{5}{2} p_{\alpha} \mathbf{v}_{0}+\varepsilon_{\alpha} n_{\alpha} \mathbf{v}_{0}\right)-\mathbf{F}_{\alpha}^{(1)} \cdot \rho_{\alpha} \mathbf{v}_{0}\right]\right\} \\
& +\frac{\partial}{\partial \mathbf{r}} \cdot\left\{\frac{1}{2} \rho_{\alpha} v_{0}^{2} \mathbf{v}_{0}+\frac{5}{2} p_{\alpha} \mathbf{v}_{0}+\varepsilon_{\alpha} n_{\alpha} \mathbf{v}_{0}-\tau_{\alpha}\left[\frac{\partial}{\partial t}\left(\frac{1}{2} \rho_{\alpha} v_{0}^{2} \mathbf{v}_{0}+\frac{5}{2} p_{\alpha} \mathbf{v}_{0}+\varepsilon_{\alpha} n_{\alpha} \mathbf{v}_{0}\right)\right.\right. \\
& +\frac{\partial}{\partial \mathbf{r}} \cdot\left(\frac{1}{2} \rho_{\alpha} v_{0}^{2} \mathbf{v}_{0} \mathbf{v}_{0}+\frac{7}{2} p_{\alpha} \mathbf{v}_{0} \mathbf{v}_{0}+\frac{1}{2} p_{\alpha} v_{0}^{2} \overrightarrow{\mathrm{I}}+\frac{5}{2} \frac{p_{\alpha}^{2}}{\rho_{\alpha}} \overrightarrow{\mathrm{I}}+\varepsilon_{\alpha} n_{\alpha} \mathbf{v}_{0} \mathbf{v}_{0}+\varepsilon_{\alpha} \frac{p_{\alpha}}{m_{\alpha}} \overrightarrow{\mathrm{I}}\right) \\
& -\rho_{\alpha} \mathbf{F}_{\alpha}^{(1)} \cdot \mathbf{v}_{0} \mathbf{v}_{0}-p_{\alpha} \mathbf{F}_{\alpha}^{(1)} \cdot \overrightarrow{\mathrm{I}}-\frac{1}{2} \rho_{\alpha} v_{0}^{2} \mathbf{F}_{\alpha}^{(1)}-\frac{3}{2} \mathbf{F}_{\alpha}^{(1)} p_{\alpha}-\frac{\rho_{\alpha} v_{0}^{2}}{2} \frac{q_{\alpha}}{m_{\alpha}}\left[\mathbf{v}_{0} \times \mathbf{B}\right] \\
& \left.\left.-\frac{5}{2} p_{\alpha} \frac{q_{\alpha}}{m_{\alpha}}\left[\mathbf{v}_{0} \times \mathbf{B}\right]-\varepsilon_{\alpha} n_{\alpha} \frac{q_{\alpha}}{m_{\alpha}}\left[\mathbf{v}_{0} \times \mathbf{B}\right]-\varepsilon_{\alpha} n_{\alpha} \mathbf{F}_{\alpha}^{(1)}\right]\right\} \\
& -\left\{\rho_{\alpha} \mathbf{F}_{\alpha}^{(1)} \cdot \mathbf{v}_{0}-\tau_{\alpha}\left[\mathbf{F}_{\alpha}^{(1)} \cdot\left(\frac{\partial}{\partial t}\left(\rho_{\alpha} \mathbf{v}_{0}\right)+\frac{\partial}{\partial \mathbf{r}} \cdot \rho_{\alpha} \mathbf{v}_{0} \mathbf{v}_{0}+\frac{\partial}{\partial \mathbf{r}} \cdot p_{\alpha} \overrightarrow{\mathrm{I}}-\rho_{\alpha} \mathbf{F}_{\alpha}^{(1)}-q_{\alpha} n_{\alpha}\left[\mathbf{v}_{0} \times \mathbf{B}\right]\right)\right]\right\} \\
& =\int\left(\frac{m_{\alpha} v_{\alpha}^{2}}{2}+\varepsilon_{\alpha}\right) J_{\alpha}^{s t, e l} d \mathbf{v}_{\alpha}+\int\left(\frac{m_{\alpha} v_{\alpha}^{2}}{2}+\varepsilon_{\alpha}\right) J_{\alpha}^{s t, i n e l} d \mathbf{v}_{\alpha} \cdot
\end{aligned}
$$

and after summation the generalized energy equation for mixture 


$$
\begin{aligned}
& \frac{\partial}{\partial t}\left\{\frac{\rho v_{0}^{2}}{2}+\frac{3}{2} p+\sum_{\alpha} \varepsilon_{\alpha} n_{\alpha}-\sum_{\alpha} \tau_{\alpha}\left[\frac{\partial}{\partial t}\left(\frac{\rho_{\alpha} v_{0}^{2}}{2}+\frac{3}{2} p_{\alpha}+\varepsilon_{\alpha} n_{\alpha}\right)\right.\right. \\
& \left.\left.+\frac{\partial}{\partial \mathbf{r}} \cdot\left(\frac{1}{2} \rho_{\alpha} v_{0}^{2} \mathbf{v}_{0}+\frac{5}{2} p_{\alpha} \mathbf{v}_{0}+\varepsilon_{\alpha} n_{\alpha} \mathbf{v}_{0}\right)-\mathbf{F}_{\alpha}^{(1)} \cdot \rho_{\alpha} \mathbf{v}_{0}\right]\right\} \\
& +\frac{\partial}{\partial \mathbf{r}} \cdot\left\{\frac{1}{2} \rho v_{0}^{2} \mathbf{v}_{0}+\frac{5}{2} p \mathbf{v}_{0}+\mathbf{v}_{0} \sum_{\alpha} \varepsilon_{\alpha} n_{\alpha}-\sum_{\alpha} \tau_{\alpha}\left[\frac { \partial } { \partial t } \left(\frac{1}{2} \rho_{\alpha} v_{0}^{2} \mathbf{v}_{0}\right.\right.\right. \\
& \left.+\frac{5}{2} p_{\alpha} \mathbf{v}_{0}+\varepsilon_{\alpha} n_{\alpha} \mathbf{v}_{0}\right)+\frac{\partial}{\partial \mathbf{r}} \cdot\left(\frac{1}{2} \rho_{\alpha} v_{0}^{2} \mathbf{v}_{0} \mathbf{v}_{0}+\frac{7}{2} p_{\alpha} \mathbf{v}_{0} \mathbf{v}_{0}+\frac{1}{2} p_{\alpha} v_{0}^{2} \overrightarrow{\mathrm{I}}\right. \\
& \left.+\frac{5}{2} \frac{p_{\alpha}^{2}}{\rho_{\alpha}} \overrightarrow{\mathrm{I}}+\varepsilon_{\alpha} n_{\alpha} \mathbf{v}_{0} \mathbf{v}_{0}+\varepsilon_{\alpha} \frac{p_{\alpha}}{m_{\alpha}} \overrightarrow{\mathrm{I}}\right)-\rho_{\alpha} \mathbf{F}_{\alpha}^{(1)} \cdot \mathbf{v}_{0} \mathbf{v}_{0}-p_{\alpha} \mathbf{F}_{\alpha}^{(1)} \cdot \overrightarrow{\mathrm{I}} \\
& -\frac{1}{2} \rho_{\alpha} v_{0}^{2} \mathbf{F}_{\alpha}^{(1)}-\frac{3}{2} \mathbf{F}_{\alpha}^{(1)} p_{\alpha}-\frac{\rho_{\alpha} v_{0}^{2}}{2} \frac{q_{\alpha}}{m_{\alpha}}\left[\mathbf{v}_{0} \times \mathbf{B}\right]-\frac{5}{2} p_{\alpha} \frac{q_{\alpha}}{m_{\alpha}}\left[\mathbf{v}_{0} \times \mathbf{B}\right] \\
& \left.\left.-\varepsilon_{\alpha} n_{\alpha} \frac{q_{\alpha}}{m_{\alpha}}\left[\mathbf{v}_{0} \times \mathbf{B}\right]-\varepsilon_{\alpha} n_{\alpha} \mathbf{F}_{\alpha}^{(1)}\right]\right\}-\mathbf{v}_{0} \cdot \sum_{\alpha} \rho_{\alpha} \mathbf{F}_{\alpha}^{(1)} \\
& +\sum_{\alpha} \tau_{\alpha} \mathbf{F}_{\alpha}^{(1)} \cdot\left[\frac{\partial}{\partial t}\left(\rho_{\alpha} \mathbf{v}_{0}\right)+\frac{\partial}{\partial \mathbf{r}} \cdot \rho_{\alpha} \mathbf{v}_{0} \mathbf{v}_{0}+\frac{\partial}{\partial \mathbf{r}} \cdot p_{\alpha} \overrightarrow{\mathrm{I}}-\rho_{\alpha} \mathbf{F}_{\alpha}^{(1)}-q_{\alpha} n_{\alpha}\left[\mathbf{v}_{0} \times \mathbf{B}\right]\right]=0 .
\end{aligned}
$$

Here $\mathbf{F}_{\alpha}^{(1)}$ are the forces of the non-magnetic origin, B - magnetic induction, $\overrightarrow{\mathrm{I}}$ - unit tensor, $q_{\alpha}$ charge of the $\alpha$-component particle, $p_{\alpha}$ - static pressure for $\alpha$-component, $\varepsilon_{\alpha}$ - internal energy for the particles of $\alpha$ - component, $\mathbf{v}_{0}$ - hydrodynamic velocity for mixture. For calculations in the selfconsistent electro-magnetic field the system of non-local Maxwell equations should be added.

\section{Quantum Solitons in the Self Consistent Electric Field}

Here we intend to demonstrate the appearance of quantum solitons in plasma objects. Let us introduce the coordinate system moving along the positive direction of $x$ - axis in ID space with velocity $C=u_{0}$ equal to phase velocity of considering quantum object

$$
\xi=x-C t
$$

Taking into account the De Broglie relation we should wait that the group velocity $u_{g}$ is equal $2 u_{0}$. In moving coordinate system all dependent hydrodynamic values are function of $(\xi, t)$.We investigate the possibility of the quantum object formation of the soliton type. For this solution there is no explicit dependence on time for coordinate system moving with the phase velocity $u_{0}$. Write down the system of equations (2.1) - (2.6) for the two component mixture of charged particles without taking into account the component's internal energy in the dimensionless form, where dimensionless symbols are marked by tildes.

In the following we intend to obtain the soliton's type of solution of the generalized hydrodynamic equations for plasma in the self consistent electrical field. All elements of possible formation like quantum soliton should move with the same translational velocity. Then the system of GHE consists from the generalized Poisson equation reflecting the effects of the charge and the charge flux perturbations, two continuity equations for positive and negative species (in particular, for ion and electron components), one motion equation and two energy equations for ion and electron components. This system of six equations for non-stationary $1 \mathrm{D}$ case can be written in the form [1 - 3]:

(Poisson equation)

$$
\frac{\partial^{2} \phi}{\partial x^{2}}=-4 \pi e\left\{\left[n_{i}-\tau_{i}\left(\frac{\partial n_{i}}{\partial t}+\frac{\partial}{\partial x}\left(n_{i} u\right)\right)\right]-\left[n_{e}-\tau_{e}\left(\frac{\partial n_{e}}{\partial t}+\frac{\partial}{\partial x}\left(n_{e} u\right)\right)\right]\right\}
$$


(continuity equation for positive ion component)

$$
\frac{\partial}{\partial t}\left\{\rho_{i}-\tau_{i}\left[\frac{\partial \rho_{i}}{\partial t}+\frac{\partial}{\partial x}\left(\rho_{i} u\right)\right]\right\}+\frac{\partial}{\partial x}\left\{\rho_{i} u-\tau_{i}\left[\frac{\partial}{\partial t}\left(\rho_{i} u\right)+\frac{\partial}{\partial x}\left(\rho_{i} u^{2}\right)+\frac{\partial p_{i}}{\partial x}-\rho_{i} F_{i}\right]\right\}
$$

(continuity equation for electron component)

$$
\frac{\partial}{\partial t}\left\{\rho_{e}-\tau_{e}\left[\frac{\partial \rho_{e}}{\partial t}+\frac{\partial}{\partial x}\left(\rho_{e} u\right)\right]\right\}+\frac{\partial}{\partial x}\left\{\rho_{e} u-\tau_{e}\left[\frac{\partial}{\partial t}\left(\rho_{e} u\right)+\frac{\partial}{\partial x}\left(\rho_{e} u^{2}\right)+\frac{\partial p_{e}}{\partial x}-\rho_{e} F_{e}\right]\right\}
$$

(momentum equation)

$$
\begin{aligned}
& \frac{\partial}{\partial t}\left\{\rho u-\tau_{i}\left[\frac{\partial}{\partial t}\left(\rho_{i} u\right)+\frac{\partial}{\partial x}\left(p_{i}+\rho_{i} u^{2}\right)-\rho_{i} F_{i}\right]-\tau_{e}\left[\frac{\partial}{\partial t}\left(\rho_{e} u\right)+\frac{\partial}{\partial x}\left(p_{e}+\rho_{e} u^{2}\right)-\rho_{e} F_{e}\right]\right\} \\
& -\rho_{i} F_{i}-\rho_{e} F_{e}+F_{i} \tau_{i}\left(\frac{\partial \rho_{i}}{\partial t}+\frac{\partial}{\partial x}\left(\rho_{i} u\right)\right)+F_{e} \tau_{e}\left(\frac{\partial \rho_{e}}{\partial t}+\frac{\partial}{\partial x}\left(\rho_{e} u\right)\right) \\
& +\frac{\partial}{\partial x}\left\{\begin{array}{l}
\rho u^{2}+p-\tau_{i}\left[\frac{\partial}{\partial t}\left(\rho_{i} u^{2}+p_{i}\right)+\frac{\partial}{\partial x}\left(\rho_{i} u^{3}+3 p_{i} u\right)-2 \rho_{i} u F_{i}\right] \\
-\tau_{e}\left[\frac{\partial}{\partial t}\left(\rho_{e} u^{2}+p_{e}\right)+\frac{\partial}{\partial x}\left(\rho_{e} u^{3}+3 p_{e} u\right)\right]-2 \rho_{e} u F_{e}
\end{array}\right\}=0
\end{aligned}
$$

(energy equation for positive ion component)

$$
\begin{aligned}
& \frac{\partial}{\partial t}\left\{\rho_{i} u^{2}+3 p_{i}-\tau_{i}\left[\frac{\partial}{\partial t}\left(\rho_{i} u^{2}+3 p_{i}\right)+\frac{\partial}{\partial x}\left(\rho_{i} u^{3}+5 p_{i} u\right)-2 \rho_{i} F_{i} u\right]\right\} \\
& +\frac{\partial}{\partial x}\left\{\rho_{i} u^{3}+5 p_{i} u-\tau_{i}\left[\frac{\partial}{\partial t}\left(\rho_{i} u^{3}+5 p_{i} u\right)+\frac{\partial}{\partial x}\left(\rho_{i} u^{4}+8 p_{i} u^{2}+5 \frac{p_{i}^{2}}{\rho_{i}}\right)-F_{i}\left(3 \rho_{i} u^{2}+5 p_{i}\right)\right]\right\} \\
& -2 u \rho_{i} F_{i}+2 \tau_{i} F_{i}\left[\frac{\partial}{\partial t}\left(\rho_{i} u\right)+\frac{\partial}{\partial x}\left(\rho_{i} u^{2}+p_{i}\right)-\rho_{i} F_{i}\right]=-\frac{p_{i}-p_{e}}{\tau_{e i}}
\end{aligned}
$$

(energy equation for electron component)

$$
\begin{aligned}
& \frac{\partial}{\partial t}\left\{\rho_{e} u^{2}+3 p_{e}-\tau_{e}\left[\frac{\partial}{\partial t}\left(\rho_{e} u^{2}+3 p_{e}\right)+\frac{\partial}{\partial x}\left(\rho_{e} u^{3}+5 p_{e} u\right)-2 \rho_{e} F_{e} u\right]\right\} \\
& +\frac{\partial}{\partial x}\left\{\rho_{e} u^{3}+5 p_{e} u-\tau_{e}\left[\frac{\partial}{\partial t}\left(\rho_{e} u^{3}+5 p_{e} u\right)+\frac{\partial}{\partial x}\left(\rho_{e} u^{4}+8 p_{e} u^{2}+5 \frac{p_{e}^{2}}{\rho_{e}}\right)-F_{e}\left(3 \rho_{e} u^{2}+5 p_{e}\right)\right]\right\} \\
& -2 u \rho_{e} F_{e}+2 \tau_{e} F_{e}\left[\frac{\partial}{\partial t}\left(\rho_{e} u\right)+\frac{\partial}{\partial x}\left(\rho_{e} u^{2}+p_{e}\right)-\rho_{e} F_{e}\right]=-\frac{p_{e}-p_{i}}{\tau_{e i}}
\end{aligned}
$$

where $u$ is translational velocity of the quantum object, $\phi$ - scalar potential, $n_{i}$ and $n_{e}$ are the number density of the charged species, $F_{i}$ and $F_{e}$ are the forces acting on the unit mass of ion and electron. The $\tau_{i}, \tau_{i}$ and $\tau_{e i}$ approximations are used in the forms (1.25), (1.26).

The following formulae are valid for acting forces

$$
F_{i}=-\frac{e}{m_{i}} \frac{\partial \phi}{\partial x}, F_{e}=\frac{e}{m_{e}} \frac{\partial \phi}{\partial x}
$$

Let us transform the system of equations (3.2) - (3.7) into dimensionless form using the scales

$$
u_{0}, x_{0}=\frac{\hbar}{m_{e}} \frac{1}{u_{0}}, \phi_{0}=\frac{m_{e}}{e} u_{0}^{2}, \rho_{0}=\frac{m_{e}^{4}}{4 \pi \hbar^{2} e^{2}} u_{0}^{4}, p_{0}=\rho_{0} u_{0}^{2}=\frac{m_{e}^{4}}{4 \pi \hbar^{2} e^{2}} u_{0}^{6}
$$

From the introduced scales only two parameters are independent - the phase velocity $u_{0}$ of the quantum object, and external parameter $H$, which is proportional to Plank constant $\hbar$ and in general case should be inserted in the scale relation as $x_{0}=H /\left(m_{e} u_{0}\right)=n \hbar /\left(m_{e} u_{0}\right)$. It leads to exchange in all 
scales $\hbar \leftrightarrow H$. But the value $v^{q u}=\hbar / m_{e}$ has the dimension $\left[\mathrm{cm}^{2} / \mathrm{s}\right]$ and can be titled as quantum viscosity, $v^{q u}=1.1577 \mathrm{~cm}^{2} / \mathrm{s}$. Of course in principal the electron mass can be replaced in scales by mass of other particles with the negative charge. From this point of view the obtained solutions have the universal character defined only by Cauchy conditions.

Comments to the following system of six dimensionless ordinary non-linear equations $(3.10)-(3.16)$ :

1. Every equation from the system is of the second order and needs two conditions. The problem belongs to the class of Cauchy problems.

2. In comparison with the Schrödinger theory connected with behavior of the wave function, no special conditions are applied for dependent variables including the domain of the solution existing. This domain is defined automatically in the process of the numerical solution of the concrete variant of calculations.

$$
\begin{aligned}
& \frac{\partial^{2} \tilde{\psi}}{\partial \tilde{\xi}^{2}}=-\left\{\frac{m_{e}}{m_{i}}\left[\tilde{\rho}_{i}-\frac{1}{\tilde{u}^{2}} \frac{m_{e}}{m_{i}}\left(-\frac{\partial \tilde{\rho}_{i}}{\partial \tilde{\xi}}+\frac{\partial}{\partial \tilde{\xi}}\left(\tilde{\rho}_{i} \tilde{u}\right)\right)\right]-\left[\tilde{\rho}_{e}-\frac{1}{\tilde{u}^{2}}\left(-\frac{\partial \tilde{\rho}_{e}}{\partial \tilde{\xi}}+\frac{\partial}{\partial \tilde{\xi}}\left(\tilde{\rho}_{e} \tilde{u}\right)\right)\right]\right\}, \\
& \frac{\partial \tilde{\rho}_{i}}{\partial \tilde{\xi}}-\frac{\partial}{\partial \tilde{\xi}}\left(\tilde{\rho}_{i} \tilde{u}\right)+\frac{m_{e}}{m_{i}} \frac{\partial}{\partial \tilde{\xi}}\left\{\frac{1}{\tilde{u}^{2}}\left[\frac{\partial}{\partial \tilde{\xi}}\left(\tilde{p}_{i}+\tilde{\rho}_{i}+\tilde{\rho}_{i} \tilde{u}^{2}-2 \tilde{\rho}_{i} \tilde{u}\right)+\frac{m_{e}}{m_{i}} \tilde{\rho}_{i} \frac{\partial \tilde{\psi}}{\partial \tilde{\xi}}\right]\right\}=0, \\
& \frac{\partial \tilde{\rho}_{e}}{\partial \tilde{\xi}}-\frac{\partial}{\partial \tilde{\xi}}\left(\tilde{\rho}_{e} \tilde{u}\right)+\frac{\partial}{\partial \tilde{\xi}}\left\{\frac{1}{\tilde{u}^{2}}\left[\frac{\partial}{\partial \tilde{\xi}}\left(\tilde{p}_{e}+\tilde{\rho}_{e}+\tilde{\rho}_{e} \tilde{u}^{2}-2 \tilde{\rho}_{e} \tilde{u}\right)+\tilde{\rho}_{e} \frac{\partial \tilde{\psi}}{\partial \tilde{\xi}}\right]\right\}=0, \\
& \frac{\partial}{\partial \tilde{\xi}}\left\{\left(\tilde{\rho}_{i}+\tilde{\rho}_{e}\right) \tilde{u}^{2}+\left(\tilde{p}_{i}+\tilde{p}_{e}\right)-\left(\tilde{\rho}_{i}+\tilde{\rho}_{e}\right) \tilde{u}\right\}+ \\
& +\frac{\partial}{\partial \tilde{\xi}}\left\{\begin{array}{l}
\frac{1}{\tilde{u}^{2}} \frac{m_{e}}{m_{i}}\left[\frac{\partial}{\partial \tilde{\xi}}\left(2 \tilde{p}_{i}+2 \tilde{\rho}_{i} \tilde{u}^{2}-\tilde{\rho}_{i} \tilde{u}-\tilde{\rho}_{i} \tilde{u}^{3}-3 \tilde{p}_{i} \tilde{u}\right)+\tilde{\rho}_{i} \frac{m_{e}}{m_{i}} \frac{\partial \tilde{\psi}}{\partial \tilde{\xi}}\right] \\
+\frac{1}{\tilde{u}^{2}}\left[\frac{\partial}{\partial \tilde{\xi}}\left(2 \tilde{p}_{e}+2 \tilde{\rho}_{e} \tilde{u}^{2}-\tilde{\rho}_{e} \tilde{u}-\tilde{\rho}_{e} \tilde{u}^{3}-3 \tilde{p} \tilde{u}\right)-\tilde{\rho}_{e} \frac{\partial \tilde{\psi}}{\partial \tilde{\xi}}\right]
\end{array}\right\} \\
& +\tilde{\rho}_{i} \frac{m_{e}}{m_{i}} \frac{\partial \tilde{\psi}}{\partial \tilde{\xi}}-\tilde{\rho}_{e} \frac{\partial \tilde{\psi}}{\partial \tilde{\xi}}-\frac{\partial \tilde{\psi}}{\partial \tilde{\xi}} \frac{1}{\tilde{u}^{2}}\left(\frac{m_{e}}{m_{i}}\right)^{2}\left(-\frac{\partial \tilde{\rho}_{i}}{\partial \tilde{\xi}}+\frac{\partial}{\partial \tilde{\xi}}\left(\tilde{\rho}_{i} \tilde{u}\right)\right) \\
& +\frac{\partial \tilde{\psi}}{\partial \tilde{\xi}} \frac{1}{\tilde{u}^{2}}\left(-\frac{\partial \tilde{\rho}_{e}}{\partial \tilde{\xi}}+\frac{\partial}{\partial \tilde{\xi}}\left(\tilde{\rho}_{e} \tilde{u}\right)\right)-2 \frac{\partial}{\partial \tilde{\xi}}\left\{\frac{1}{\tilde{u}} \frac{\partial \tilde{\psi}}{\partial \tilde{\xi}}\left[\left(\frac{m_{e}}{m_{i}}\right)^{2} \tilde{\rho}_{i}-\tilde{\rho}_{e}\right]\right\}=0 \\
& \frac{\partial}{\partial \tilde{\xi}}\left\{\tilde{\rho}_{i} \tilde{u}^{3}+5 \tilde{p}_{i} \tilde{u}-\tilde{\rho}_{i} \tilde{u}^{2}-3 \tilde{p}_{i}\right\} \\
& +\frac{\partial}{\partial \tilde{\xi}}\left\{\begin{array}{l}
\frac{1}{u^{2}} \frac{m_{e}}{m_{i}}\left[\frac{\partial}{\partial \tilde{\xi}}\left(2 \tilde{\rho}_{i} \tilde{u}^{3}+10 \tilde{p} \tilde{u}_{i}-\tilde{\rho}_{i} \tilde{u}^{4}-8 \tilde{p}_{i} \tilde{u}^{2}-5 \frac{\tilde{p}_{i}^{2}}{\tilde{\rho}_{i}}-\tilde{\rho}_{i} \tilde{u}^{2}-3 \tilde{p}_{i}\right)\right] \\
+\frac{m_{e}}{m_{i}} \frac{\partial \tilde{\psi}}{\partial \tilde{\xi}}\left(2 \tilde{\rho}_{i} \tilde{u}-3 \tilde{\rho}_{i} \tilde{u}^{2}-5 \tilde{p}_{i}\right)
\end{array}\right\} \\
& +2 \frac{m_{e}}{m_{i}} \tilde{\rho} \tilde{u} \frac{\partial \tilde{\psi}}{\partial \tilde{\xi}} \\
& -2 \frac{\partial \tilde{\psi}}{\partial \tilde{\xi}}\left(\frac{m_{e}}{m_{i}}\right)^{2} \frac{1}{u^{2}}\left[\frac{\partial}{\partial \tilde{\xi}}\left(\tilde{\rho}_{i} \tilde{u}^{2}+\tilde{p}_{i}-\tilde{\rho}_{i} \tilde{u}\right)+\frac{m_{e}}{m_{i}} \tilde{\rho}_{i} \frac{\partial \tilde{\psi}}{\partial \tilde{\xi}}\right]=-\left(\tilde{p}_{i}-\tilde{p}_{e}\right) \tilde{u}^{2}\left(1+\frac{m_{i}}{m_{e}}\right)
\end{aligned}
$$




$$
\begin{aligned}
& \frac{\partial}{\partial \tilde{\xi}}\left\{\tilde{\rho}_{e} \tilde{u}^{3}+5 \tilde{p} \tilde{u}-\tilde{\rho}_{e} \tilde{u}^{2}-3 \tilde{p}_{e}\right\} \\
& +\frac{\partial}{\partial \tilde{\xi}}\left\{\begin{array}{l}
\frac{1}{u^{2}}\left[\frac{\partial}{\partial \tilde{\xi}}\left(2 \tilde{\rho} \tilde{u}_{e} \tilde{u}^{3}+10 \tilde{p} \tilde{u}-\tilde{\rho}_{e} \tilde{u}^{4}-8 \tilde{p} \tilde{u}_{e}^{2}-5 \frac{\tilde{p}_{e}^{2}}{\tilde{\rho}_{e}}-\tilde{\rho}_{e} \tilde{u}^{2}-3 \tilde{p}_{e}\right)\right] \\
-\frac{\partial \tilde{\psi}}{\partial \tilde{\xi}}\left(2 \tilde{\rho}_{e} \tilde{u}-3 \tilde{\rho} \tilde{u}_{e}^{2}-5 \tilde{p}_{e}\right)
\end{array}\right\} \\
& -2 \tilde{\rho} e \tilde{u} \frac{\partial \tilde{\psi}}{\partial \tilde{\xi}} \\
& +2 \frac{\partial \tilde{\psi}}{\partial \tilde{\xi}} \frac{1}{u^{2}}\left[\frac{\partial}{\partial \tilde{\xi}}\left(\tilde{\rho} \tilde{\rho}_{e} \tilde{u}^{2}+\tilde{p}_{e}-\tilde{\rho}_{e} \tilde{u}\right)+\tilde{\rho}_{e} \frac{\partial \tilde{\psi}}{\partial \tilde{\xi}}\right]=-\left(\tilde{p}_{e}-\tilde{p}_{i}\right) \tilde{u}^{2}\left(1+\frac{m_{i}}{m_{e}}\right)
\end{aligned}
$$

Analogs of the BGK approximations were used for the energy collision integral approximation.

\section{Some Results of Mathematical Modeling}

The system of generalized quantum hydrodynamic equations (3.10) - (3.15) have the great possibilities of mathematical modeling as result of changing of twelve Cauchy conditions describing the character features of initial perturbations which lead to the soliton formation. The following figures reflect some results of calculations realized according to the system of equations (3.10) - (3.15) with the help of Maple 9. The following notations on figures are used: r- density $\tilde{\rho}_{i}$ (solid black line), s- density $\tilde{\rho}_{e}$ (solid line), u- velocity $\tilde{u}$ (dashed line), $\mathrm{p}$ - pressure $\tilde{p}_{i}$ (black dash dotted line), $q$ - pressure $\tilde{p}_{e}$ (dash dotted line) and $\mathrm{v}$ - self consistent potential $\tilde{\phi}$. Explanations placed under all following figures, Maple program contains Maple's notations - for example the expression $D(u)(0)=0$ means in usual notations $\frac{\partial \tilde{u}}{\partial \tilde{\xi}}(0)=0$, independent variable $t$ responds to $\tilde{\xi}$. We begin with investigation of the problem of principle significance - is it possible after a perturbation (defined by Cauchy conditions) to obtain the quantum object of the soliton's kind as result of the self-organization of ionized matter? In the case of the positive answer, what is the origin of existence of this stable object? By the way the mentioned questions belong to the typical problem in the theory of the ball lightning. With this aim let us consider the initial perturbations

$$
\begin{aligned}
& v(0)=1, r(0)=1, s(0)=1, u(0)=1, p(0)=1, q(0)=.95, \\
& D(v)(0)=0, D(r)(0)=0, D(s)(0)=0, D(u)(0)=0, D(p)(0)=0, D(q)(0)=0
\end{aligned}
$$

in the mixture of positive and negative ions of equal masses if the pressure $\tilde{p}_{i}(0)$ of positive particles is larger than $\tilde{p}_{e}(0)$ of the negative ones (for the variant under consideration $\mathbf{p}(\mathbf{0})=\mathbf{1}, \mathbf{q}(\mathbf{0})=. \mathbf{9 5}$ ). The following Figures $1-3$ reflect the result of solution of Eqs. (3.10)-(3.15). 


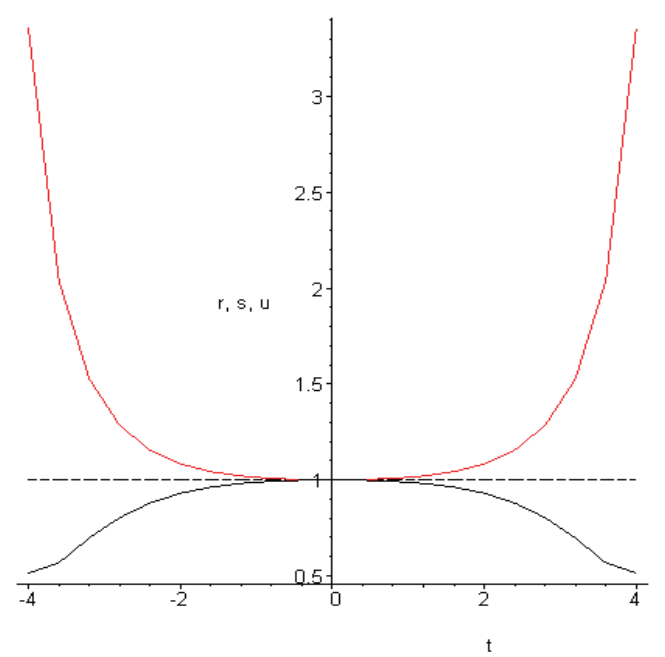

Figure 4.1. $\mathrm{r}$ - density $\tilde{\rho}_{i}$ (low line), u- velocity $\tilde{u}$ (dashed line), s- density $\tilde{\rho}_{e}$.

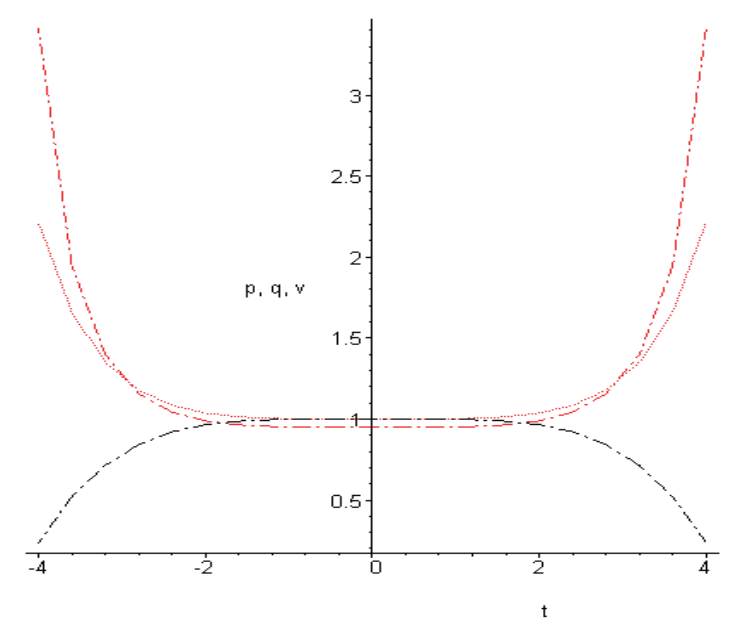

Figure 4.2. p - pressure $\tilde{p}_{i}$ (low line), $q$ - pressure $\tilde{p}_{e}, \mathrm{v}$ - self-consistent potential $\tilde{\psi}$ in quantum soliton (solid line).
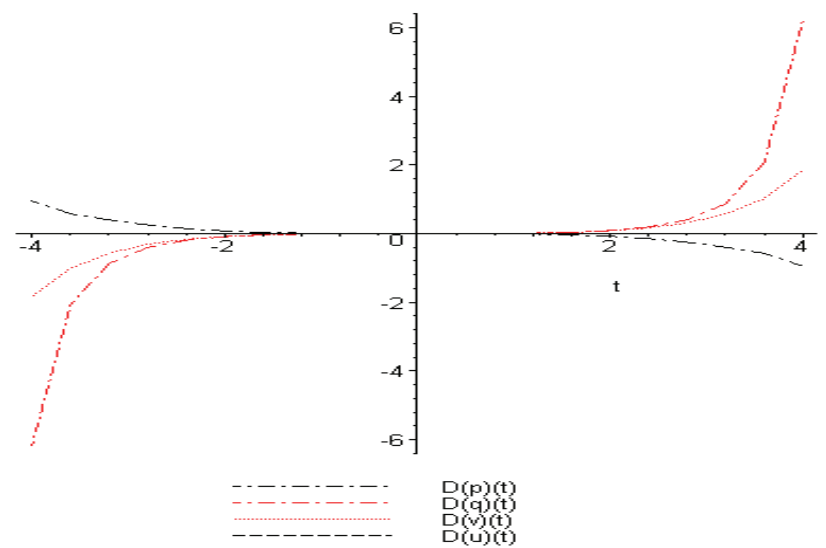

Figure 4.3. The derivative of pressure of the positive component $\partial \tilde{p}_{i} / \partial \tilde{\xi}$ and negative component $\partial \tilde{p}_{e} / \partial \tilde{\xi}$, the derivative of the self-consistent potential $\partial \tilde{\phi} / \partial \tilde{\xi}$ in quantum soliton. 
Fig. 4.1 displays the quantum object placed in bounded region of $1 \mathrm{D}$ space, all parts of this object are moving with the same velocity. It's Important to underline that no special boundary conditions were used for this and all following cases. Then this soliton is product of the self-organization of ionized matter. Figures 4.2, 4.3 contain the answer for formulated above questions about stability of the object. Really the object is restricted by negative shell. The derivative $\partial \tilde{\psi} / \partial \tilde{\xi}$ is proportional to the selfconsistent forces acting on the positive and negative parts of the soliton. Consider for example the right side of soliton. The self consistent force of the electrical origin compresses the positive part of this soliton and provokes the movement of the negative part along the positive direction of the $\tilde{\xi}$-axis $(t-$ axis in nomination of Fig. 4.1). But the increasing of quantum pressure prevent to destruction of soliton. Therefore the stability of the quantum object is result of the self-consistent influence of electric potential and quantum pressures. Interesting to notice that stability can be also achieved if soliton has positive shell and negative kernel but $\tilde{p}_{i}(0)<\tilde{p}_{e}(0)$. Figures 4.4, 4.5 displays the typical quantum object placed in bounded region of $1 \mathrm{D}$ space, all parts of this object are moving with the same velocity. Figures 4.4, 4.5 reflect the following Cauchy conditions (written in Maple notations):

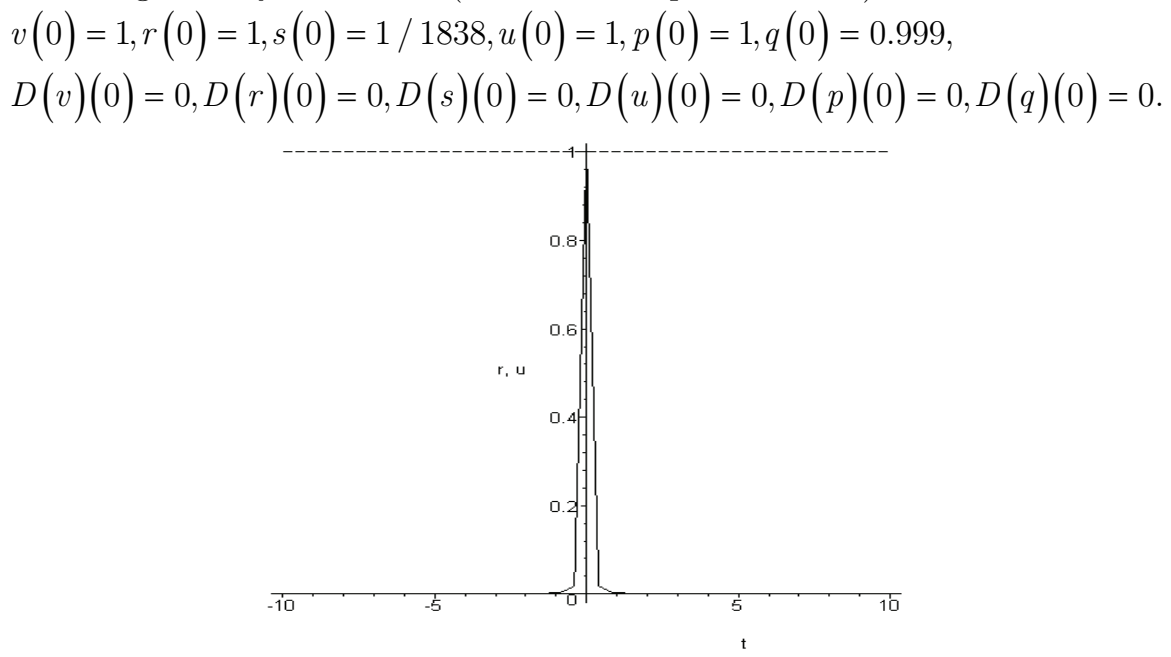

Figure 4.4. r- density $\tilde{\rho}_{i}$, u- velocity $\tilde{u}$ in quantum soliton.

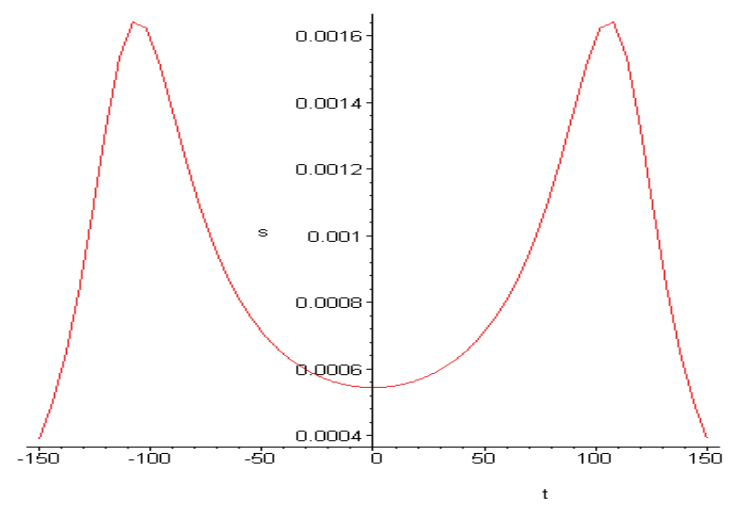

Figure 4.5. s- density $\tilde{\rho}_{e}$ in quantum soliton.

\section{Mathematical Modeling of the Ball Lightning Structure}

The ball lightning (BL) problem might be considered as the unique one. Over the past two centuries several hundreds of hypotheses of ball lightning structure have been proposed [13], and now from time to time new hypotheses are still emerging. No reason to discuss these theories. Moreover we intend to proof the theorem - all the lightning's balls models based on local physics are wrong in principal. At the 
same time the efforts are initiated to obtain the artificial ball lightning. Often they are undertaken in the hope of fortune, of occasional success. These attempts are inspired by the apparent simplicity of ball lightning creating in nature, where it is most likely generated by a discharge of a linear lightning. However the success has not been yet achieved on that way (see for example [14 - 16]). The cause of this situation is obvious - no underlying theory.

We have now the sea of observational data. These data were statistically treated and were checked for reliability. The main results of this procedure are:

1) data observed in different parts of the Earth which are in good agreement with each other [13, 17 $22]$,

2) all these data describe one and the same natural phenomenon [23-28].

The following characteristics of ball lightning might be considered as the main ones:

1. Its ability to liberate up to $10^{9} \mathrm{~J}$ of energy in a matter of several tens of seconds. Nevertheless it is not an upper limit, because the Tunguska phenomenon can be treated as a giant BL explosion.

2. The peculiar character of its movement (absence of convection, movement against the wind, floating along conductors).

3. The lifetime up to several minutes, quiet dying or destruction with explosion.

4. The mechanism of its luminosity in the visible range of spectrum (light power 10-1000 W, colour red, orange, silver, rainbow, possibility of fast colour changing, stability of emission power during its lifetime).

5. Absence of heat emission, and burns at close contact.

6. The presence or absence of noise and odour, accompanying its appearance.

7. Its ability to penetrate through obstacles (glasses, nets) with or without damaging them.

8. Its capability to change form, split into fragments and penetrate through chinks.

9. Its ability to lift heavy objects and to perform mechanical work.

The purpose of the following presentation is to apply the methods of non-local physics to the study of long-lived (quasi-stationary) solitary plasma objects, which we will further call also as plasmoids. Introduction to the definition of the requirement of solitary of the object is fundamental, because it allows you to separate the phenomenon from the lights of St. Elmo-glow near individual objects, which is observed in stormy weather at high electric field. St. Elmo's lights are attached to objects, and are treated as a corona discharge glow in the vicinity of these objects. The term "plasmoid" is not unambiguous; in the literature, a plasmoid is often understood as a limited configuration of magnetic fields and plasma. Next, we will see that for a long-lived plasma formation, generally speaking, no external or self-consistent magnetic field is required. The use of the term "ball lightning" is even less preferable because the shape of an object can vary from spherical (or not correct spherical) to pearshaped.

Ball lightning is a serious danger to the observer. In 1753, Georg Richman, a full member of the St. Petersburg Academy of Sciences, was killed by a ball lightning strike. He created a device to study atmospheric electricity. When at the next meeting, Richman heard that the storm was coming; he urgently went home with the engraver to capture the phenomenon. During the experiment, the blueorange ball flew out of the device and hit the scientist directly on the forehead. There was a deafening roar. Richman fell dead, and the engraver was stunned and knocked down. He later described what happened. On the forehead of the scientist was a little dark crimson stain, his clothes were parched, and shoes torn. Door shattered into splinters, and the door blew off its hinges. Later inspection of a scene was made personally by M. V. Lomonosov.

There is a reason to believe that the death of Gagarin was due to a collision his aircraft with atmospheric plasmoid, [3].

The author of the article personally watched the ball lightning (diameter about 7-10 cm) which flew during a thunderstorm at the cottage from the electrical outlet and slowly moved through the room went into the chimney of the fireplace.

There are many reports collected and analysed today on observations of long-lived large-scale plasmoids in atmosphere and under water $[29,30]$. These events were reported by military sailors, pilots and radar specialists. Typical characteristics and parameters of these plasmoids are the follows:

- Diameter 3-10 m

- Altitude 10-20 km

- Depth 0- $10 \mathrm{~km}$ 
- Cruise velocity in air 2 - 4 kmps

- Cruise velocity in water $300 \mathrm{kmph}$

- Life time 0.1 - 10 hours.

Many of these plasmoids are invisible in radio-frequency range for external probing EM radiation. For example, radar couldn't record some of these plasmoids. There is a strong electromagnetic impact of these plasmoids on board electronic equipment arranged on flight or sea vehicles. This long-lived plasmoid has a high electric surface potential which is up to $10 \mathrm{kV}$. So, this plasmoid is charged (nonneutral) plasma formation. It is revealed that aerodynamic drag of charged plasmoid is very small in air and water flows. So, it can move against a wind and a water flow. The artificial primary plasmoid can be divided in two or three secondary plasmoids. There is also an opposite process of primary small plasmoids conglomeration to a single large secondary plasmoid.

In recent years, there have been reports of giant electrical discharges in the upper atmosphere at altitudes from 30 to 150 kilometers, which are often identified with unidentified flying objects (UFO). Moreover, there are reports $[29,30]$ about underwater UFO in the oceans. The appearance of underwater plasmoids with a diameter of three to ten meters is associated with electric discharges that occur when the tectonic plates are shifted.

The number of appearances of ball lightning, not directly related to the storm, is small. According to Barry, 90\% of occurrences were observed during thunderstorms. It is not surprising that experimenters trying to reproduce the ball lightning in the laboratory use water as one of the electrodes. In particular, A. I. Egorov and S. I. Stepanov [31] describe a device that allows to obtain luminous plasmoids artificial ball lightning - with high reproducibility. Plasmoids are obtained as a result of a high - voltage discharge in the air, one electrode is solid, the other electrode is the surface of the water. The measuring equipment was created for studying the life time and electrical properties of plasmoids. A significant part of the charge is concentrated on the glowing shell of the plasmoid.

A. G. Oreshko [32] reports on the experimental production of spherical plasmoids. The core of plasmoids has an excessive negative charge, and the outer spherical layer has, on the contrary, an excess positive charge. For us the mentioned experimental works are interesting by the fact that the excess peripheral charge of plasmoids can be positive or negative depending on the organization of the experiment.

What is the origin of all difficulties with the creation of the adequate BL theory?

1. The BL theory should be a particular case of the corresponding quantum nonlocal soliton theory.

2. The application of the BL transport equations should lead to Cauchy problems but not to boundary problems.

3. The BL theory should reflect the separation effects of charges in BL.

4. It means that the creation of the BL theory is equal to creation of the atom structure calculation with simultaneous self-consistent consideration nucleus + electron shell.

5. FNE responds to these demands.

Let us now turn to the mathematical modeling of plasmoids based on the methods of non-local physics. We apply FNE for the BL structure calculation. We write down FNE (2.1) - (2.6) in the spherical coordinate system for the plasma object in the rest (hydrodynamic velocity $\mathbf{v}_{0}=0$ ) for the one dimensional non-stationary case.

The system of the one dimensional non-stationary equations for a many species charged objects: - continuity equation

$$
\frac{\partial}{\partial t}\left[\rho_{\alpha}-\tau_{\alpha} \frac{\partial \rho_{\alpha}}{\partial t}\right]-\frac{1}{r^{2}} \frac{\partial}{\partial r}\left[r^{2} \tau_{\alpha}\left(q_{\alpha} \frac{\partial \psi}{\partial r}+\frac{\partial p_{\alpha}}{\partial r}\right)\right]=0
$$

- momentum equation, $\mathbf{e}_{r}$ projection

$$
\begin{aligned}
& -\frac{\partial}{\partial t}\left\{\tau_{\alpha}\left[\frac{\partial p_{\alpha}}{\partial r}+q_{\alpha} \frac{\partial \psi}{\partial r}\right]\right\}+\frac{q_{\alpha}}{\rho_{\alpha}} \frac{\partial \psi}{\partial r}\left[\rho_{\alpha}-\tau_{\alpha}\left(\frac{\partial \rho_{\alpha}}{\partial t}\right)\right] \\
& +\frac{\partial p_{\alpha}}{\partial r}-\frac{\partial}{\partial r}\left(\tau_{\alpha} \frac{\partial p_{\alpha}}{\partial t}\right)=\int m_{\alpha} v_{\alpha r} J_{\alpha}^{s t, e l} d \mathbf{v}_{\alpha}+\int m_{\alpha} v_{\alpha r} J_{\alpha}^{s t, \text { inel }} d \mathbf{v}_{\alpha},
\end{aligned}
$$


- energy equation

$$
\begin{aligned}
& \frac{\partial}{\partial t}\left\{\varepsilon n_{\alpha}+\frac{3}{2} p_{\alpha}-\tau_{\alpha} \frac{\partial}{\partial t}\left(\varepsilon_{\alpha} n_{\alpha}+\frac{3}{2} p_{\alpha}\right)\right\}+\tau_{\alpha} \frac{q_{\alpha}}{\rho_{\alpha}} \frac{\partial \psi}{\partial r}\left(\frac{\partial p_{\alpha}}{\partial r}+q_{\alpha} \frac{\partial \psi}{\partial r}\right) \\
& -\frac{1}{r^{2}} \frac{\partial}{\partial r}\left\{\tau_{\alpha} r^{2}\left[\frac{\partial}{\partial r}\left[\frac{p_{\alpha}}{\rho_{\alpha}}\left(\frac{5}{2} p_{\alpha}+\varepsilon_{\alpha} n_{\alpha}\right)\right]+\left(\frac{5}{2} p_{\alpha}+\varepsilon_{\alpha} n_{\alpha}\right) \frac{q_{\alpha}}{\rho_{\alpha}} \frac{\partial \psi}{\partial r}\right]\right\} \\
& =\int\left(\frac{m_{\alpha} v_{\alpha}^{2}}{2}+\varepsilon_{\alpha}\right) J_{\alpha}^{s t, e l} d \mathbf{v}_{\alpha} .
\end{aligned}
$$

After multiplication continuity equation (5.1) by $\varepsilon_{\alpha}$ we find

$$
\frac{\partial}{\partial t}\left[\varepsilon_{\alpha} n_{\alpha}-\tau_{\alpha} \frac{\partial \varepsilon_{\alpha} n_{\alpha}}{\partial t}\right]-\frac{1}{r^{2}} \frac{\partial}{\partial r}\left[r^{2} \tau_{\alpha}\left(\frac{q_{\alpha}}{m_{\alpha}} \frac{\partial \psi}{\partial r}+\frac{\varepsilon_{\alpha}}{m_{\alpha}} \frac{\partial p_{\alpha}}{\partial r}\right)\right]=0
$$

Taking into account (5.4) we find from (5.3)

$$
\begin{aligned}
& \frac{3}{2} \frac{\partial}{\partial t}\left\{p_{\alpha}-\tau_{\alpha} \frac{\partial}{\partial t} p_{\alpha}\right\}+\tau_{\alpha} \frac{q_{\alpha}}{\rho_{\alpha}} \frac{\partial \psi}{\partial r}\left(\frac{\partial p_{\alpha}}{\partial r}+q_{\alpha} \frac{\partial \psi}{\partial r}\right) \\
& -\frac{5}{2} \frac{1}{r^{2}} \frac{\partial}{\partial r}\left\{\tau_{\alpha} r^{2}\left[\frac{\partial}{\partial r}\left(\frac{p_{\alpha}^{2}}{\rho_{\alpha}}\right)+p_{\alpha} \frac{q_{\alpha}}{\rho_{\alpha}} \frac{\partial \psi}{\partial r}\right]\right\}=\int \frac{m_{\alpha} v_{\alpha}^{2}}{2} J_{\alpha}^{s t, e l} d \mathbf{v}_{\alpha}
\end{aligned}
$$

The consideration of the stationary case leads to the following simplification of the system (5.1), (5.2), (5.5):

$$
\begin{gathered}
q_{\alpha} \frac{\partial \psi}{\partial r}+\frac{\partial p_{\alpha}}{\partial r}=0 \\
q_{\alpha} \frac{\partial \psi}{\partial r}+\frac{\partial p_{\alpha}}{\partial r}=\int m_{\alpha} v_{\alpha r} J_{\alpha}^{s t, e l} d \mathbf{v}_{\alpha}+\int m_{\alpha} v_{\alpha r} J_{\alpha}^{s t, i n e l} d \mathbf{v}_{\alpha}, \\
\tau_{\alpha} \frac{q_{\alpha}}{\rho_{\alpha}} \frac{\partial \psi}{\partial r}\left(\frac{\partial p_{\alpha}}{\partial r}+q_{\alpha} \frac{\partial \psi}{\partial r}\right)-\frac{5}{2} \frac{1}{r^{2}} \frac{\partial}{\partial r}\left\{\tau_{\alpha} r^{2}\left[\frac{\partial}{\partial r}\left(\frac{p_{\alpha}^{2}}{\rho_{\alpha}}\right)+p_{\alpha} \frac{q_{\alpha}}{\rho_{\alpha}} \frac{\partial \psi}{\partial r}\right]\right\}=\int \frac{m_{\alpha} v_{\alpha}^{2}}{2} J_{\alpha}^{s t, e l} d \mathbf{v}_{\alpha} .
\end{gathered}
$$

To the corresponding system of equations the Poisson equation should be added

$$
\frac{1}{r^{2}} \frac{\partial}{\partial r}\left(r^{2} \frac{\partial \psi}{\partial r}\right)=-4 \pi \sum_{\alpha} q_{\alpha}
$$

where $\psi$ - scalar electric potential and $q_{\alpha}$ is a value of charge (with the corresponding sign) per the unit of volume; $q_{\alpha}= \pm e n_{\alpha}$, $e$ is the absolute electron charge. For the two species mixture we can use Poisson equation

$$
\frac{1}{r^{2}} \frac{\partial}{\partial r}\left(r^{2} \frac{\partial \psi}{\partial r}\right)=-4 \pi e\left[n_{i}-n_{e}\right]
$$

where $e$ is the absolute electron charge. Using (5.5) we find from (5.8)

or

$$
-\frac{5}{2} \frac{1}{r^{2}} \frac{\partial}{\partial r}\left\{\tau_{\alpha} r^{2}\left[\frac{\partial}{\partial r}\left(\frac{p_{\alpha}^{2}}{\rho_{\alpha}}\right)+p_{\alpha} \frac{q_{\alpha}}{\rho_{\alpha}} \frac{\partial \psi}{\partial r}\right]\right\}=\int \frac{m_{\alpha} v_{\alpha}^{2}}{2} J_{\alpha}^{s t, e l} d \mathbf{v}_{\alpha} .
$$

$$
-\frac{5}{2} \frac{1}{r^{2}} \frac{\partial}{\partial r}\left\{\tau_{\alpha} r^{2}\left[\frac{p_{\alpha}}{\rho_{\alpha}} \frac{\partial p_{\alpha}}{\partial r}+p_{\alpha} \frac{\partial}{\partial r}\left(\frac{p_{\alpha}}{\rho_{\alpha}}\right)+p_{\alpha} \frac{q_{\alpha}}{\rho_{\alpha}} \frac{\partial \psi}{\partial r}\right]\right\}=\int \frac{m_{\alpha} v_{\alpha}^{2}}{2} J_{\alpha}^{s t, e l} d \mathbf{v}_{\alpha} .
$$

or using (5.6)

$$
-\frac{5}{2} \frac{1}{r^{2}} \frac{\partial}{\partial r}\left\{\tau_{\alpha} r^{2} p_{\alpha} \frac{\partial}{\partial r}\left(\frac{p_{\alpha}}{\rho_{\alpha}}\right)\right\}=\int \frac{m_{\alpha} v_{\alpha}^{2}}{2} J_{\alpha}^{s t, e l} d \mathbf{v}_{\alpha} .
$$

Then we have the following system of equations: 


$$
\begin{gathered}
q_{\alpha} \frac{\partial \psi}{\partial r}+\frac{\partial p_{\alpha}}{\partial r}=0 \\
\frac{1}{r^{2}} \frac{\partial}{\partial r}\left(r^{2} \frac{\partial \psi}{\partial r}\right)=-4 \pi \sum_{\alpha} q_{\alpha}, \\
\frac{1}{r^{2}} \frac{\partial}{\partial r}\left\{\tau_{\alpha} r^{2} p_{\alpha} \frac{\partial}{\partial r}\left(\frac{p_{\alpha}}{\rho_{\alpha}}\right)\right\}=-\frac{2}{5} \int \frac{m_{\alpha} v_{\alpha}^{2}}{2} J_{\alpha}^{s t, e l} d \mathbf{v}_{\alpha} .
\end{gathered}
$$

For the integral collision terms we can use an analog of the BGK approximation. We reach the system of equations (indexes " $\mathrm{i}$ " and "e" can be treated also as corresponding to internal (nucleus) and external (shell) areas):

$$
\begin{gathered}
\frac{5}{2} \frac{\partial}{\partial r}\left\{r^{2} p_{i} \tau_{i} \frac{\partial}{\partial r}\left(\frac{p_{i}}{n_{i}}\right)\right\}=-m_{i} \frac{p_{i}-p_{e}}{\tau_{e i}} r^{2} \\
\frac{5}{2} \frac{\partial}{\partial r}\left\{r^{2} p_{e} \tau_{e} \frac{\partial}{\partial r}\left(\frac{p_{e}}{n_{e}}\right)\right\}=-m_{e} \frac{p_{e}-p_{i}}{\tau_{e i}} r^{2} \\
e n_{i} \frac{\partial \psi}{\partial r}+\frac{\partial p_{i}}{\partial r}=0 \\
-e n_{e} \frac{\partial \psi}{\partial r}+\frac{\partial p_{e}}{\partial r}=0 \\
\frac{\partial}{\partial r}\left(r^{2} \frac{\partial \psi}{\partial r}\right)=-4 \pi e\left[n_{i}-n_{e}\right] r^{2} .
\end{gathered}
$$

It is interesting to note a certain similarity of three equations $(5.17),(5.18)$ and (5.21).

In the frame of local physics $\left(\tau_{e}=0, \tau_{i}=0, \tau_{e i}=0\right)$ we have from $(5.17)-(5.21)$ only trivial solutions, namely

$$
p_{e}=p_{i}, n_{i}=n_{e}, \psi=\text { const }
$$

Equations (5.17) - (5.21) can be rewritten in the hydrodynamic dimensionless form using the scales: $n_{0}, p_{0}, \psi_{0}, r_{0}$. Equations (3.25) - (3.27) take the form

$$
\begin{gathered}
-e \frac{n_{0} \psi_{0}}{p_{0}} \tilde{n}_{e} \frac{\partial \tilde{\psi}}{\partial \tilde{r}}+\frac{\partial \tilde{p}_{e}}{\partial \tilde{r}}=0, \\
e \frac{n_{0} \psi_{0}}{p_{0}} \tilde{n}_{i} \frac{\partial \tilde{\psi}}{\partial \tilde{r}}+\frac{\partial \tilde{p}_{i}}{\partial \tilde{r}}=0, \\
\frac{\psi_{0}}{4 \pi e n_{0} r_{0}^{2}} \frac{1}{\tilde{r}^{2}} \frac{\partial}{\partial \tilde{r}}\left(\tilde{r}^{2} \frac{\partial \tilde{\psi}}{\partial \tilde{r}}\right)=-\left[\tilde{n}_{i}-\tilde{n}_{e}\right]
\end{gathered}
$$

Let us introduce the additional condition

and the dimensionless coefficient

$$
e \frac{n_{0} \psi_{0}}{p_{0}}=1
$$

We find

$$
A=\frac{\psi_{0}}{4 \pi e r_{0}^{2} n_{0}}=\frac{\psi_{0}^{2}}{4 \pi r_{0}^{2} p_{0}}
$$

$$
\begin{gathered}
-\tilde{n}_{e} \frac{\partial \tilde{\psi}}{\partial \tilde{r}}+\frac{\partial \tilde{p}_{e}}{\partial \tilde{r}}=0 \\
\tilde{n}_{i} \frac{\partial \tilde{\psi}}{\partial \tilde{r}}+\frac{\partial \tilde{p}_{i}}{\partial \tilde{r}}=0
\end{gathered}
$$




$$
\begin{gathered}
A \frac{1}{\tilde{r}^{2}} \frac{\partial}{\partial \tilde{r}}\left(\tilde{r}^{2} \frac{\partial \tilde{\psi}}{\partial \tilde{r}}\right)=-\left[\tilde{n}_{i}-\tilde{n}_{e}\right], \\
\frac{5}{2} \tau_{i} \tau_{e i} \frac{p_{0}}{m_{i} n_{0} r_{0}^{2}} \frac{\partial}{\partial \tilde{r}}\left\{\tilde{r}^{2} \tilde{p}_{i} \frac{\partial}{\partial \tilde{r}}\left(\frac{\tilde{p}_{i}}{\tilde{n}_{i}}\right)\right\}=-\left(\tilde{p}_{i}-\tilde{p}_{e}\right) \tilde{r}^{2}, \\
\frac{5}{2} \tau_{e} \tau_{e i} \frac{p_{0}}{m_{e} n_{0} r_{0}^{2}} \frac{\partial}{\partial \tilde{r}}\left\{\tilde{r}^{2} \tilde{p}_{e} \frac{\partial}{\partial \tilde{r}}\left(\frac{\tilde{p}_{e}}{\tilde{n}_{e}}\right)\right\}=-\left(\tilde{p}_{e}-\tilde{p}_{i}\right) \tilde{r}^{2},
\end{gathered}
$$

Let us introduce the nonlocal kinematic viscosities

$$
\begin{aligned}
& v_{i}=\frac{5}{2} \tau_{e i} \frac{p_{0}}{m_{i} n_{0}}, \\
& v_{e}=\frac{5}{2} \tau_{e i} \frac{p_{0}}{m_{e} n_{0}}
\end{aligned}
$$

and nonlocal Reynolds numbers using the definitions

$$
\begin{aligned}
& \operatorname{Re}_{i}=L V_{i} / v_{i}, \\
& \operatorname{Re}_{e}=L V_{e} / v_{e},
\end{aligned}
$$

where the linear scale is $L=r_{0}$, character velocities $V_{i}=r_{0} / \tau_{i}, V_{e}=r_{0} / \tau_{e}$.

Then equations (5.31), (5.32) take the hydrodynamic form

$$
\begin{aligned}
& \frac{1}{\operatorname{Re}_{i}} \frac{\partial}{\partial \tilde{r}}\left[\tilde{r}^{2} \tilde{p}_{i} \frac{\partial}{\partial \tilde{r}}\left(\frac{\tilde{p}_{i}}{\tilde{n}_{i}}\right)\right]=-\tilde{r}^{2}\left(\tilde{p}_{i}-\tilde{p}_{e}\right), \\
& \frac{1}{\operatorname{Re}_{e}} \frac{\partial}{\partial \tilde{r}}\left[\tilde{r}^{2} \tilde{p}_{e} \frac{\partial}{\partial \tilde{r}}\left(\frac{\tilde{p}_{e}}{\tilde{n}_{e}}\right)\right]=-\tilde{r}^{2}\left(\tilde{p}_{e}-\tilde{p}_{i}\right) .
\end{aligned}
$$

We find the system of equations of the hydrodynamic type:

$$
\begin{gathered}
-\tilde{n}_{e} \frac{\partial \tilde{\psi}}{\partial \tilde{r}}+\frac{\partial \tilde{p}_{e}}{\partial \tilde{r}}=0 \\
\tilde{n}_{i} \frac{\partial \tilde{\psi}}{\partial \tilde{r}}+\frac{\partial \tilde{p}_{i}}{\partial \tilde{r}}=0 \\
A \frac{1}{\tilde{r}^{2}} \frac{\partial}{\partial \tilde{r}}\left(\tilde{r}^{2} \frac{\partial \tilde{\psi}}{\partial \tilde{r}}\right)=-\left[\tilde{n}_{i}-\tilde{n}_{e}\right] . \\
\frac{1}{\operatorname{Re}_{i}} \frac{\partial}{\partial \tilde{r}}\left[\tilde{r}^{2} \tilde{p}_{i} \frac{\partial}{\partial \tilde{r}}\left(\frac{\tilde{p}_{i}}{\tilde{n}_{i}}\right)\right]=-\tilde{r}^{2}\left(\tilde{p}_{i}-\tilde{p}_{e}\right), \\
\frac{1}{\operatorname{Re}_{e}} \frac{\partial}{\partial \tilde{r}}\left[\tilde{r}^{2} \tilde{p}_{e} \frac{\partial}{\partial \tilde{r}}\left(\frac{\tilde{p}_{e}}{\tilde{n}_{e}}\right)\right]=-\tilde{r}^{2}\left(\tilde{p}_{e}-\tilde{p}_{i}\right)
\end{gathered}
$$

with hydrodynamic parameters $A, \operatorname{Re}_{i}, \operatorname{Re}_{e}$.

We demonstrate the characteristic features of solutions of equations (5.39) - (5.43). The results of mathematical modeling are obtained with the help of the Maple application software package (can be used versions of Maple 9 or older versions). The system of generalized hydrodynamic equations (5.39) (5.43) has wide possibilities in mathematical modeling as a result of changes in eight Cauchy conditions that determine the characteristics of the primary disturbances in the physical system, leading to the formation of a plasmoid. In the following figures, the Maple notation is used, namely: $\mathrm{r}-\tilde{n}_{i}$ (dimensionless number density of positively charged particles); s- $\tilde{n}_{e}$ (absolute dimensionless value of the number density of negatively charged particles); p-pressure $\tilde{p}_{i}$; q-pressure $\tilde{p}_{e}$ and v-self-consistent potential $\tilde{\psi}$. Explanations are placed under each figure, while using program Maple notation-for 
example, the expression $D(\mathrm{v})(0)=0$ means in the usual notation $\frac{\partial \tilde{\psi}}{\partial \tilde{r}}(0)=0$, the independent variable t corresponds $\tilde{r}, C \leftrightarrow \operatorname{Re}_{i}, E \leftrightarrow \operatorname{Re}_{e}$.

We will start with the study of the problem of fundamental importance - is it possible after the primary perturbation determined by Cauchy conditions to obtain an object of the soliton type located in a limited area of space and being a product of self-organization of ionized matter? To this end, we introduce a system of the following primary disturbances

(Option 1):

$$
\begin{aligned}
& \mathbf{p}(\mathbf{0})=0.9, \mathbf{q}(\mathbf{0})=\mathbf{1}, \mathbf{v}(\mathbf{0})=\mathbf{1}, \mathrm{r}(\mathbf{0})=\mathbf{1}, \mathrm{s}(\mathbf{0})=\mathbf{1}, \mathrm{D}(\mathbf{p})(\mathbf{0})=\mathbf{0}, \mathrm{D}(\mathbf{q})(\mathbf{0})=\mathbf{0}, \mathrm{D}(\mathrm{v})(\mathbf{0})=\mathbf{0}, \\
& \mathrm{D}(\mathrm{r})(\mathbf{0})=\mathbf{0}, \mathrm{D}(\mathrm{s})(\mathbf{0})=\mathbf{0} ; A=\operatorname{Re}_{e}=\operatorname{Re}_{i}=1
\end{aligned}
$$

(Option 2):

$$
\begin{aligned}
& \mathrm{p}(0)=1, \mathrm{q}(0)=0.9, \mathrm{v}(0)=1, \mathrm{r}(0)=1, \mathrm{~s}(0)=1, \mathrm{D}(\mathrm{p})(0)=0, \mathrm{D}(\mathrm{q})(0)=0, \mathrm{D}(\mathrm{v})(0)=0, \\
& \mathbf{D}(\mathbf{r})(\mathbf{0})=\mathbf{0}, \mathbf{D}(\mathbf{s})(\mathbf{0})=\mathbf{0} ; A=\operatorname{Re}_{e}=\operatorname{Re}_{i}=1 \text {. }
\end{aligned}
$$

(Option 3):

$$
\begin{aligned}
& \mathbf{p}(\mathbf{0})=\mathbf{0 . 9}, \mathbf{q}(\mathbf{0})=\mathbf{1}, \mathbf{v}(\mathbf{0})=\mathbf{1}, \mathbf{r}(\mathbf{0})=\mathbf{1}, \mathrm{s}(\mathbf{0})=\mathbf{1}, \mathrm{D}(\mathbf{p})(\mathbf{0})=\mathbf{0}, \mathrm{D}(\mathbf{q})(\mathbf{0})=\mathbf{0}, \mathrm{D}(\mathbf{v})(\mathbf{0})=\mathbf{0}, \\
& \mathbf{D}(\mathbf{r})(\mathbf{0})=\mathbf{0}, \mathbf{D}(\mathrm{s})(\mathbf{0})=\mathbf{0} ; A=1, \operatorname{Re}_{e}=\operatorname{Re}_{i}=0.1
\end{aligned}
$$

(Option 4):

$$
\begin{aligned}
& \mathrm{p}(\mathbf{0})=\mathbf{1}, \mathbf{q}(\mathbf{0})=\mathbf{0 . 9}, \mathrm{v}(\mathbf{0})=\mathbf{1}, \mathrm{r}(\mathbf{0})=\mathbf{1}, \mathrm{s}(\mathbf{0})=\mathbf{1}, \mathrm{D}(\mathrm{p})(\mathbf{0})=\mathbf{0}, \mathrm{D}(\mathbf{q})(\mathbf{0})=\mathbf{0}, \mathrm{D}(\mathrm{v})(\mathbf{0})=\mathbf{0}, \\
& \mathrm{D}(\mathrm{r})(\mathbf{0})=\mathbf{0}, \mathrm{D}(\mathrm{s})(\mathbf{0})=\mathbf{0} ; A=1, \operatorname{Re}_{e}=\operatorname{Re}_{i}=0.1
\end{aligned}
$$

(Option 5):

$$
\begin{aligned}
& \mathbf{p}(\mathbf{0})=\mathbf{1}, \mathbf{q}(\mathbf{0})=\mathbf{1}, \mathbf{v}(\mathbf{0})=\mathbf{1}, \mathrm{r}(\mathbf{0})=\mathbf{1}, \mathrm{s}(\mathbf{0})=\mathbf{1}, \mathrm{D}(\mathbf{p})(\mathbf{0})=\mathbf{0}, \mathrm{D}(\mathbf{q})(\mathbf{0})=\mathbf{0}, \mathrm{D}(\mathrm{v})(\mathbf{0})=\mathbf{0}, \\
& \mathrm{D}(\mathrm{r})(\mathbf{0})=\mathbf{0}, \mathrm{D}(\mathrm{s})(\mathbf{0})=\mathbf{0} ; A=\mathrm{Re}_{e}=\mathrm{Re}_{i}=1
\end{aligned}
$$

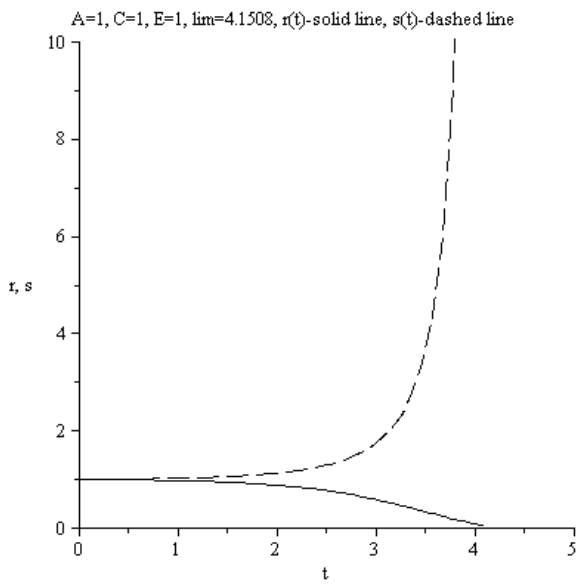

Figure 5.1. r- density $\tilde{n}_{i}$ (solid line), s- density $\tilde{n}_{e}$ (dashed line) in quantum soliton. Option 1. 


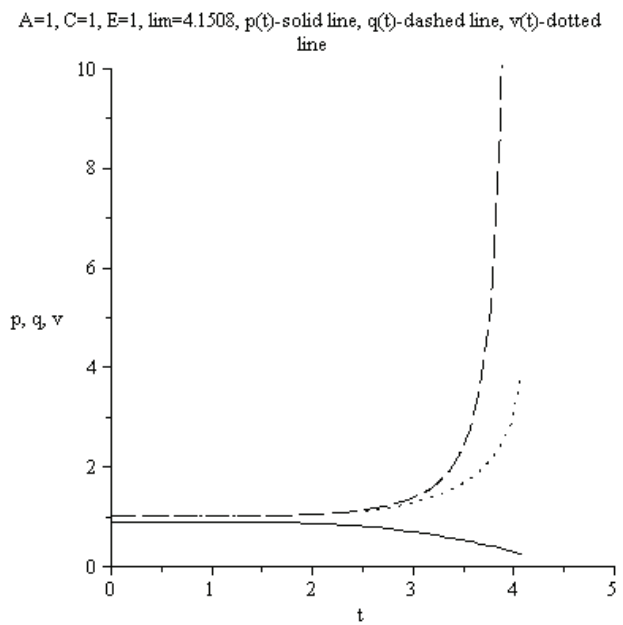

Figure 5.2. p- pressure $\tilde{p}_{i}$ (solid line), q- pressure $\tilde{p}_{e}$, (dashed line), v-potential $\tilde{\psi}$ in quantum soliton (dotted line). Option 1.

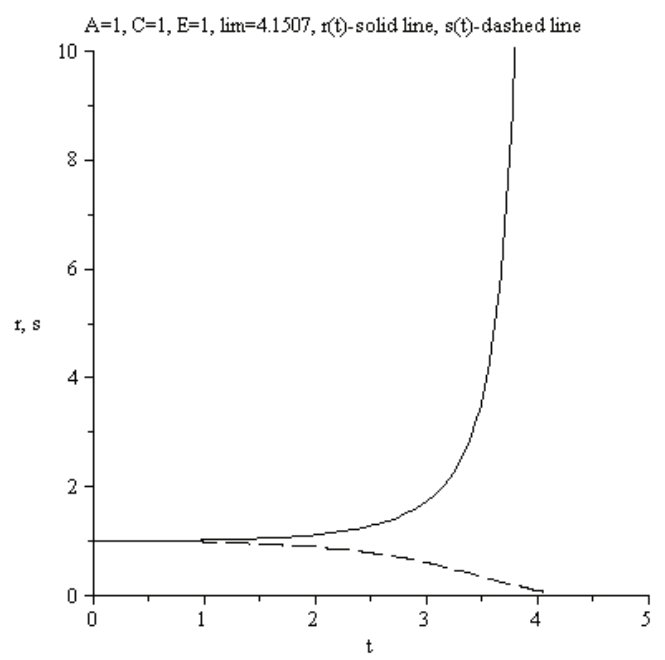

Figure 5.3. r- density $\tilde{n}_{i}$ (solid line), s- density $\tilde{n}_{e}$ (dashed line) in quantum soliton. Option 2.

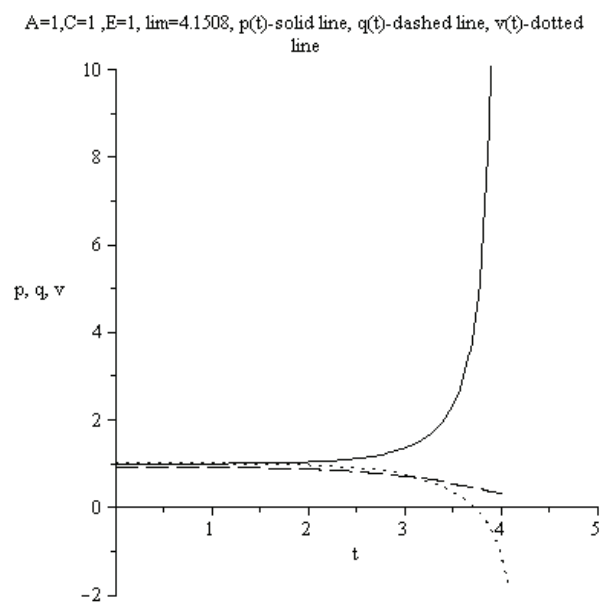

Figure 5.4. p- pressure $\tilde{p}_{i}$ (solid line), q- pressure $\tilde{p}_{e}$, (dashed line), v-potential $\tilde{\psi}$ in quantum soliton (dotted line). Option 2. 


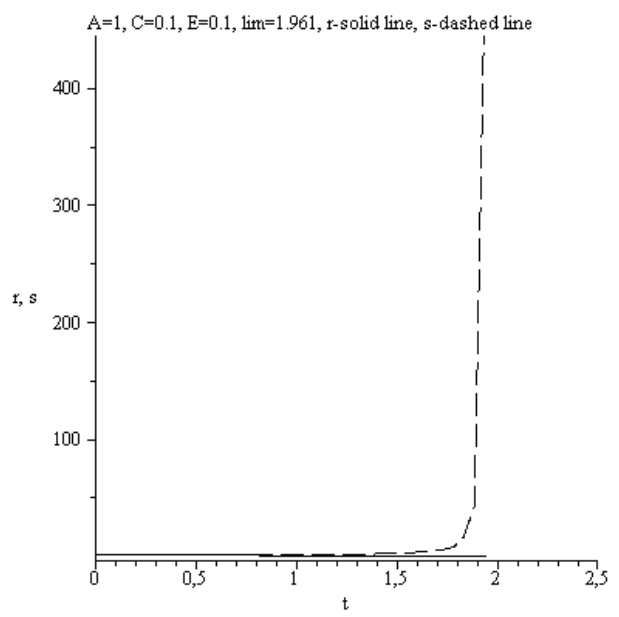

Figure 5.5. r- density $\tilde{n}_{i}$ (solid line), s- density $\tilde{n}_{e}$ (dashed line) in quantum soliton. Option 3.

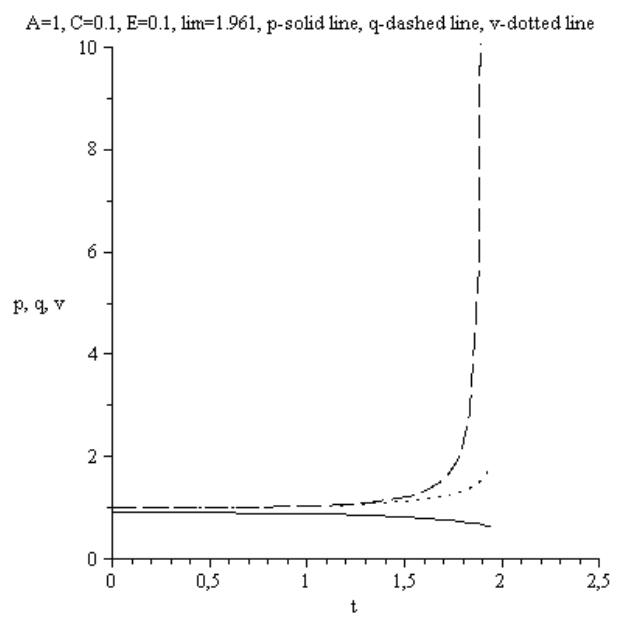

Figure 5.6. p- pressure $\tilde{p}_{i}$ (solid line), q- pressure $\tilde{p}_{e}$, (dashed line), v-potential $\tilde{\psi}$ in quantum soliton (dotted line). Option 3.

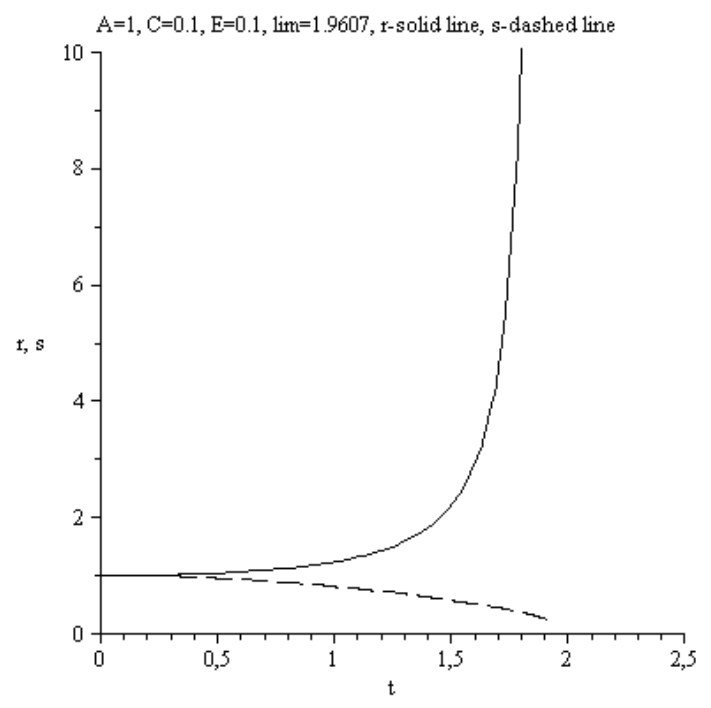

Figure 5.7. r- density $\tilde{n}_{i}$ (solid line), s- density $\tilde{n}_{e}$ (dashed line) in quantum soliton. Option 4. 


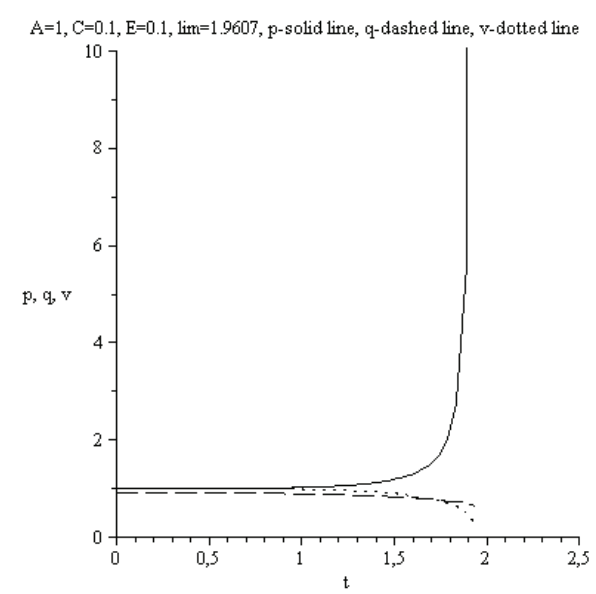

Figure 5.8. p- pressure $\tilde{p}_{i}$ (solid line), q- pressure $\tilde{p}_{e}$, (dashed line), v-potential $\tilde{\psi}$ in quantum soliton (dotted line). Option 4.

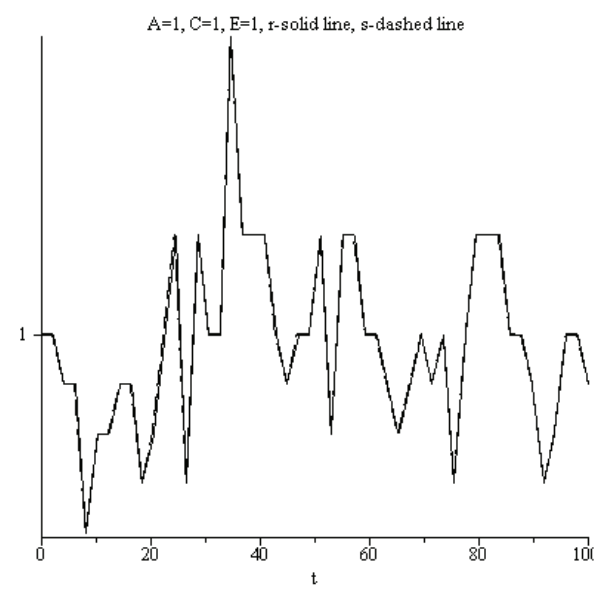

Figure 5.9. r- density $\tilde{n}_{i}$ (solid line), s- density $\tilde{n}_{e}$ (dashed line) in quantum soliton. Option 5.

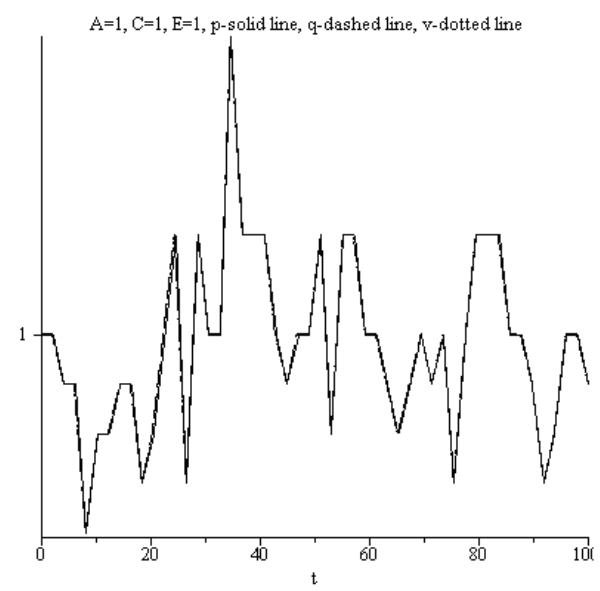

Figure 5.10. p- pressure $\tilde{p}_{i}$ (solid line), q- pressure $\tilde{p}_{e}$, (dashed line), v-potential $\tilde{\psi}$ in quantum soliton (dotted line). Option 5.

We will draw conclusions using the results of the calculations: 
1. A plasmoid is a non-equilibrium product of self-organization of ionized matter located in a limited region of space. A non-equilibrium object has an excess (in relation to the equilibrium configuration) charge of one sign and a lack of charges of another sign along the radial direction. Figures 5.1 - 5.7 show the existence of two spherical layers whose size is determined by Cauchy conditions and parameters $A, \operatorname{Re}_{i}, \operatorname{Re}_{e}$. The stability of the plasma object is achieved as a result of the equilibrium of self-consistent forces of electrostatic origin and gas-kinetic pressure of non-local origin. In this case, the outer spherical electric layer can bear a negative charge if $\tilde{p}_{i}(0)<\tilde{p}_{e}(0)$ or positive charge if $\tilde{p}_{i}(0)>\tilde{p}_{e}(0)$. As follows from the given experimental data $[31,32]$, both of these cases are implemented in practice and are determined by the specific features of the organization of the experiment.

2. If the initial gradient of pressures is absent and $\tilde{p}_{i}(0)=\tilde{p}_{e}(0)$ then calculations lead to physical natural results - plasma fluctuations (figures 5.9 and 5.10).

3. Mathematical modeling implemented in the framework of non-local physics leads to the existence of a stable plasma object even in the absence of a magnetic field.

4. In the considered theory, there is no need to use external boundary conditions. The radial size of the plasmoid is produced "automatically" in the process of solving the Cauchy problem as a domain of existence of the solution.

Let us introduce now quantization in the developed theory. The following transformations lead to quantization of the mentioned Reynolds numbers. Let us use now the discussed before the quantum adiabatic approximations for nonlocal parameters (1.33), (1.34):

$$
\begin{gathered}
\tau_{e}=\frac{n}{v_{e}} \text { for electronic shell, } \\
\tau_{i}=\frac{k}{v_{i}} \text { for nucleus, }
\end{gathered}
$$

where $n$ is shell quantum number and $k$ is nucleus quantum number. We reach the system of quantum equations:

$$
\begin{gathered}
-\tilde{n}_{e} \frac{\partial \tilde{\psi}}{\partial \tilde{r}}+\frac{\partial \tilde{p}_{e}}{\partial \tilde{r}}=0, \\
\tilde{n}_{i} \frac{\partial \tilde{\psi}}{\partial \tilde{r}}+\frac{\partial \tilde{p}_{i}}{\partial \tilde{r}}=0, \\
A \frac{1}{\tilde{r}^{2}} \frac{\partial}{\partial \tilde{r}}\left(\tilde{r}^{2} \frac{\partial \tilde{\psi}}{\partial \tilde{r}}\right)=-\left[\tilde{n}_{i}-\tilde{n}_{e}\right], \\
\frac{5}{2} \frac{\partial}{\partial \tilde{r}}\left\{\tilde{r}^{2} \tilde{p}_{i} \frac{\partial}{\partial \tilde{r}}\left(\frac{\tilde{p}_{i}}{\tilde{n}_{i}}\right)\right\}=-\frac{m_{i}}{M}\left(\tilde{p}_{i}-\tilde{p}_{e}\right) \frac{\tilde{v}_{i}}{k}\left[\frac{\tilde{v}_{e}}{n}+\frac{\tilde{v}_{i}}{k}\right] \tilde{r}^{2}, \\
\frac{5}{2} \frac{\partial}{\partial \tilde{r}}\left\{\tilde{r}^{2} \tilde{p}_{e} \frac{\partial}{\partial \tilde{r}}\left(\frac{\tilde{p}_{e}}{\tilde{n}_{e}}\right)\right\}=-\frac{m_{e}}{M}\left(\tilde{p}_{e}-\tilde{p}_{i}\right) \frac{\tilde{v}_{e}}{n}\left[\frac{\tilde{v}_{e}}{n}+\frac{\tilde{v}_{i}}{k}\right] \tilde{r}^{2},
\end{gathered}
$$

where the following notation is introduced for the mass scale:

$$
M=\frac{p_{0}}{r_{0}^{2} n_{0} v_{0}^{2}} .
$$

Comparing (5.42) and (5.49) we find

$$
\operatorname{Re}_{i}=\frac{2}{5} \frac{m_{i}}{M} \frac{\tilde{v}_{i}}{k}\left[\frac{\tilde{v}_{e}}{n}+\frac{\tilde{v}_{i}}{k}\right]
$$

and comparing (5.43) and (5.50)

$$
\operatorname{Re}_{e}=\frac{2}{5} \frac{m_{e}}{M} \frac{\tilde{v}_{e}}{n}\left[\frac{\tilde{v}_{e}}{n}+\frac{\tilde{v}_{i}}{k}\right] .
$$

Let us investigate the asymptotic of the system of equations (5.39) - (5.41) when the electron quantum numbers $k, n \rightarrow \infty$ or $\operatorname{Re}_{i} \rightarrow 0, \operatorname{Re}_{e} \rightarrow 0$. We find 


$$
\begin{gathered}
-\tilde{n}_{e} \frac{\partial \tilde{\psi}}{\partial \tilde{r}}+\frac{\partial \tilde{p}_{e}}{\partial \tilde{r}}=0, \\
\tilde{n}_{i} \frac{\partial \tilde{\psi}}{\partial \tilde{r}}+\frac{\partial \tilde{p}_{i}}{\partial \tilde{r}}=0, \\
A \frac{1}{\tilde{r}^{2}} \frac{\partial}{\partial \tilde{r}}\left(\tilde{r}^{2} \frac{\partial \tilde{\psi}}{\partial \tilde{r}}\right)=-\left[\tilde{n}_{i}-\tilde{n}_{e}\right] . \\
\frac{\partial}{\partial \tilde{r}}\left(\frac{\tilde{p}_{i}}{\tilde{n}_{i}}\right)=0, \\
\frac{\partial}{\partial \tilde{r}}\left(\frac{\tilde{p}_{e}}{\tilde{n}_{e}}\right)=0
\end{gathered}
$$

Solutions of the system (5.54) - (5.58) are defined by Cauchy conditions and only one parameter $A$. Nevertheless we prolong the transformations by integrating (5.57) and (5.58).

$$
\begin{aligned}
& \tilde{p}_{i}=P \tilde{n}_{i}, \\
& \tilde{p}_{e}=E \tilde{n}_{e} .
\end{aligned}
$$

Relation (5.59), (5.60) have the transparent physical sense. Really we have

$$
\frac{p_{\alpha}}{\rho_{\alpha}}=\text { const }
$$

or

$$
\frac{5}{2} \frac{p_{\alpha}}{\rho_{\alpha}}+\frac{\varepsilon_{\alpha}}{m_{\alpha}}=\text { const }
$$

if we use the energy equation (5.3). Relations (5.61) and (5.62) correspond to the well known laws of the energy conservation and can be considered also as equations of state. Relations (5.61) and (5.62) are equations of state for the positive and negative particles; the dimensionless parameters $E$ and $P$ are proportional to the total internal energy of the electron shell and the nucleus of the atom correspondingly. After substitution (5.60) in (5.58) and (5.59) in (5.57) we obtain

$$
\begin{gathered}
-\tilde{n}_{e} \frac{\partial \tilde{\psi}}{\partial \tilde{r}}+E \frac{\partial \tilde{n}_{e}}{\partial \tilde{r}}=0, \\
\tilde{n}_{i} \frac{\partial \tilde{\psi}}{\partial \tilde{r}}+P \frac{\partial \tilde{n}_{i}}{\partial \tilde{r}}=0
\end{gathered}
$$

or

$$
\begin{gathered}
-\frac{\partial \tilde{\psi}}{\partial \tilde{r}}+E \frac{\partial \ln \tilde{n}_{e}}{\partial \tilde{r}}=0, \\
\frac{\partial \tilde{\psi}}{\partial \tilde{r}}+P \frac{\partial \ln \tilde{n}_{i}}{\partial \tilde{r}}=0 .
\end{gathered}
$$

Then

$$
\begin{gathered}
E \ln \tilde{n}_{e}-\tilde{\psi}=B, \\
P \ln \tilde{n}_{i}+\tilde{\psi}=C, \\
\tilde{n}_{e}=\exp \left[\frac{1}{E}(B+\tilde{\psi})\right], \\
\tilde{n}_{i}=\exp \left[\frac{1}{P}(C-\tilde{\psi})\right] .
\end{gathered}
$$

Other interpretation of (5.69) and (5.70):

$$
\tilde{n}_{e}=\tilde{n}_{e 0} e^{\tilde{\psi} / E}, \tilde{n}_{i}=\tilde{n}_{i 0} e^{-\tilde{\psi} / P},
$$

where 


$$
\tilde{n}_{e 0}=\exp \frac{B}{E}, \tilde{n}_{i 0}=\exp \frac{C}{P} .
$$

Using (5.56) and (5.71), (5.72) we reach an equation

$$
A \frac{1}{\tilde{r}^{2}} \frac{\partial}{\partial \tilde{r}}\left(\tilde{r}^{2} \frac{\partial \tilde{\psi}}{\partial \tilde{r}}\right)=\exp \left[\frac{1}{E}(B+\tilde{\psi})\right]-\exp \left[\frac{1}{P}(C-\tilde{\psi})\right] .
$$

Equation (5.73) can be easily numerically integrated if the system of external parameters $A, B, C, E, P$ is known.

Let us demonstrate results of some calculations. The following system of equations was used for calculations.

$$
\begin{gathered}
A \frac{1}{\tilde{r}^{2}} \frac{\partial}{\partial \tilde{r}}\left(\tilde{r}^{2} \frac{\partial \tilde{\psi}}{\partial \tilde{r}}\right)=\tilde{n}_{e 0} e^{\psi / E}-\tilde{n}_{i 0} e^{-\psi / P} \\
-\tilde{n}_{e} \frac{\partial \tilde{\psi}}{\partial \tilde{r}}+E \frac{\partial \tilde{n}_{e}}{\partial \tilde{r}}=0 \\
\tilde{n}_{i} \frac{\partial \tilde{\psi}}{\partial \tilde{r}}+P \frac{\partial \tilde{n}_{i}}{\partial \tilde{r}}=0
\end{gathered}
$$

The pressures are

$$
\tilde{p}_{i}=P \tilde{n}_{i}, \tilde{p}_{e}=E \tilde{n}_{e} .
$$

Equations (5.75) and (5.76) can be immediately integrated (useful in analytical investigations). In particular from $(5.75)$, (5.76) follow

$$
\tilde{n}_{e}^{E} \tilde{n}_{i}^{P}=\text { const. }
$$

But the differential forms are more favorable in Maple applications. The following Maple notations on figures are used: $\mathrm{v}$ - potential $\tilde{\psi}, \mathrm{t}-$ independent variable $\tilde{r}, n_{e 0} \equiv \mathrm{H}, n_{i 0} \equiv \mathrm{Q}, \tilde{n}_{e}(\tilde{r}) \equiv \mathrm{x}(\mathrm{t})$, $\tilde{n}_{i}(\tilde{r}) \equiv \mathrm{y}(\mathrm{t})$. Explanations placed under all following figures. The system of external parameters $A, B, C, E, P$ leads to the vast possibilities of mathematical modeling. Some typical calculations are displayed on figures $5.11-5.14$.

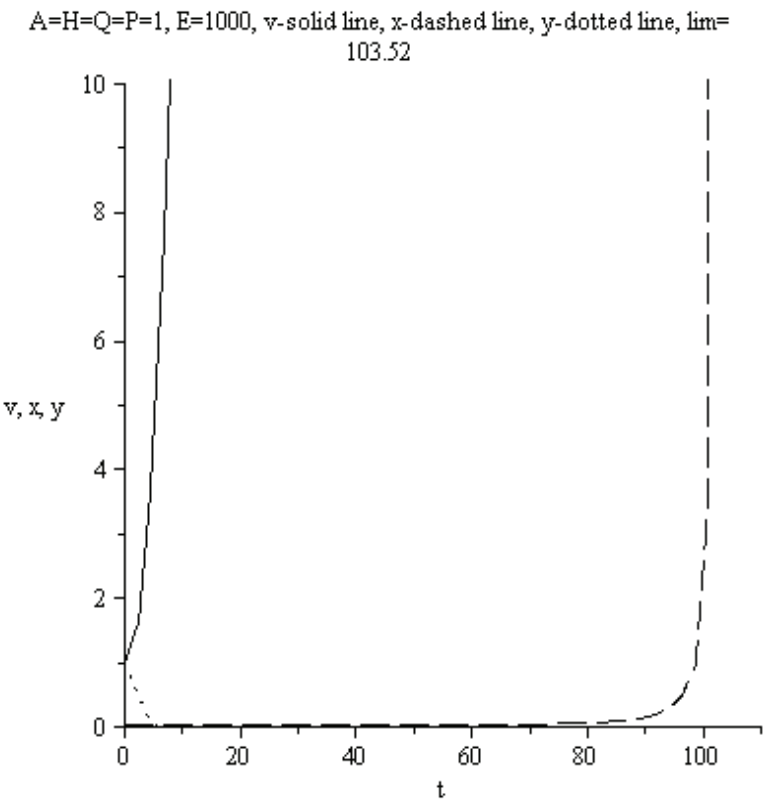

Figure 5.11. Dependence $\tilde{\psi}(\tilde{r})$ - solid line, $\tilde{n}_{e}(\tilde{r})$ - dashed line, $\tilde{n}_{i}(\tilde{r})$ - dotted line, $E=1000, \tilde{r}_{\text {lim }}=103.52$ 


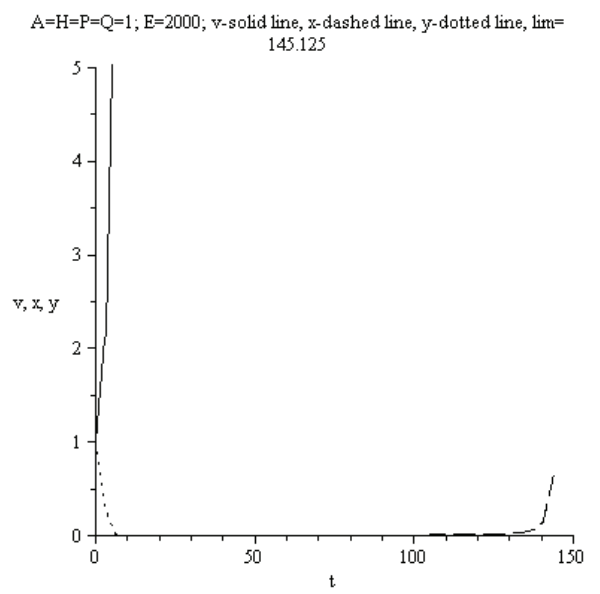

Figure 5.12. Dependence $\tilde{\psi}(\tilde{r})$ - solid line, $\tilde{n}_{e}(\tilde{r})$ - dashed line, $\tilde{n}_{i}(\tilde{r})$ - dotted line, $E=2000, \tilde{r}_{\text {lim }}=145.125$.

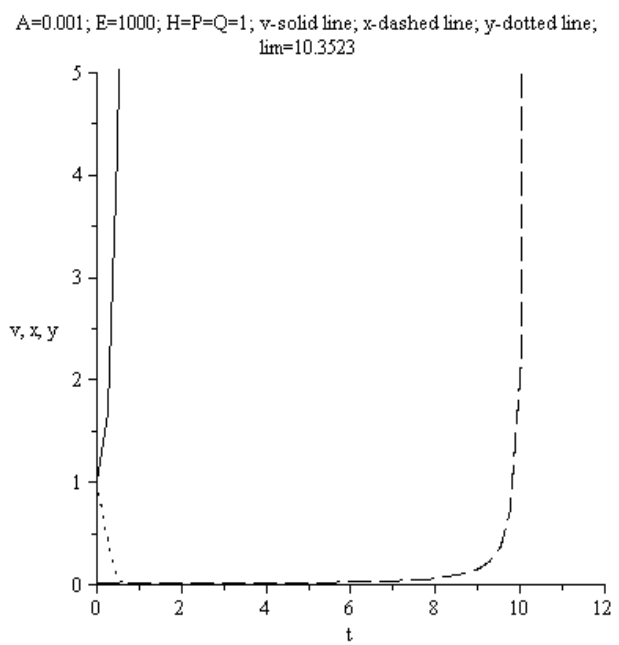

Figure 5.13. Dependence $\tilde{\psi}(\tilde{r})$-solid line, $\tilde{n}_{e}(\tilde{r})$-dashed line, $\tilde{n}_{i}(\tilde{r})$-dotted line, $E=1000, A=0.001$, $\tilde{r}_{\lim }=10.3523$

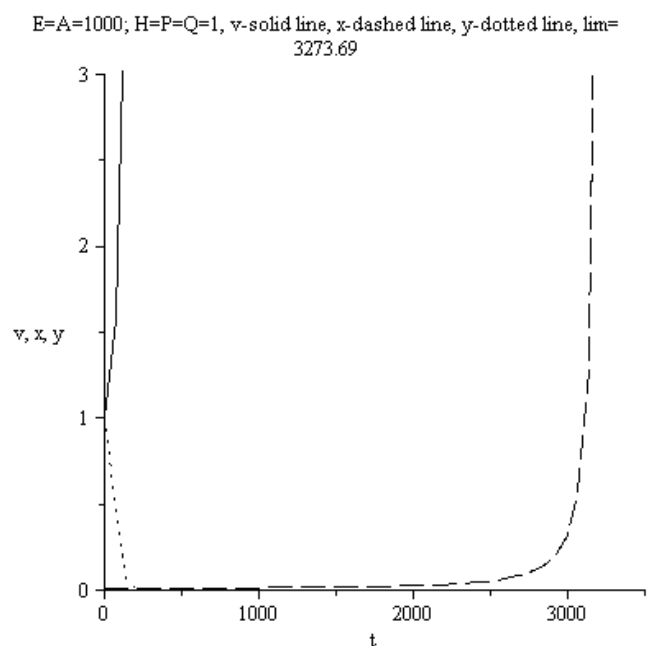

Figure 5.14. Dependence $\tilde{\psi}(\tilde{r})$ - solid line, $\tilde{n}_{e}(\tilde{r})$ - dashed line, $\tilde{n}_{i}(\tilde{r})$ - dotted line, $E=1000, A=1000$, $\tilde{r}_{\lim }=3273.69$. 
Conclusion to Item 5:

1. The domain size $\tilde{r}_{e l}<\tilde{r}_{\lim }$ is a result of the self-consistent solution. Really, the absolute electron charge $q_{e l}$ is equal to

$$
\left|q_{e l}\right|=e \int_{0}^{r_{e l}} 4 \pi r^{2} n_{e}(r) d r
$$

2. The results of calculations have the obvious physical sense. In particular, enlarging of $E$ - parameter leads to enlarging of the separation between the negative and positive charges.

3. Non-local physics reveals the possibility to calculate simultaneously the structure of the electron shell and nucleus as the self-consistent system of the separated charges placed in the finite space domain without any boundary conditions. The theory is applicable to other plasma objects including ball lightings. The corresponding solutions with the separated charges have the character of the stable solitons.

4. If the integral energy collision term can be omitted, the results of calculations do not dependent on the choice of nonlocal parameter $\tau_{\alpha}$.

5. In the nonlocal quantum hydrodynamics the particle spin is defined by the internal energy. In the case as you see the spin characteristic can be excluded from consideration.

Let us return now to the direct numerical integration of the system of equations (5.46) - (5.50), written here again with system of parameters:

$\tilde{m}_{e}=\frac{m_{e}}{M}, \tilde{m}_{i}=\frac{m_{i}}{M}, A=\frac{\psi_{0}}{4 \pi e r_{0}^{2} n_{0}}=\frac{\psi_{0}^{2}}{4 \pi r_{0}^{2} p_{0}}, M=\frac{p_{0}}{r_{0}^{2} n_{0} v_{0}^{2}}$ and scales $n_{0}, p_{0}, \psi_{0}, r_{0}, v_{0}$ with the additional condition $e \frac{n_{0} \psi_{0}}{p_{0}}=1$. Then we have tremendous possibilities for mathematical modeling. As examples in following calculations we use $\tilde{m}_{e} \tilde{v}_{e} \sim \tilde{m}_{i} \tilde{v}_{i} \sim 1$, varying only $A, k, n$.

$$
\begin{gathered}
-\tilde{n}_{e} \frac{\partial \tilde{\psi}}{\partial \tilde{r}}+\frac{\partial \tilde{p}_{e}}{\partial \tilde{r}}=0, \\
\tilde{n}_{i} \frac{\partial \tilde{\psi}}{\partial \tilde{r}}+\frac{\partial \tilde{p}_{i}}{\partial \tilde{r}}=0, \\
A \frac{1}{\tilde{r}^{2}} \frac{\partial}{\partial \tilde{r}}\left(\tilde{r}^{2} \frac{\partial \tilde{\psi}}{\partial \tilde{r}}\right)=-\left[\tilde{n}_{i}-\tilde{n}_{e}\right], \\
\frac{5}{2} \frac{\partial}{\partial \tilde{r}}\left\{\tilde{r}^{2} \tilde{p}_{i} \frac{\partial}{\partial \tilde{r}}\left(\frac{\tilde{p}_{i}}{\tilde{n}_{i}}\right)\right\}=-\frac{m_{i}}{M}\left(\tilde{p}_{i}-\tilde{p}_{e}\right) \frac{\tilde{v}_{i}}{k}\left[\frac{\tilde{v}_{e}}{n}+\frac{\tilde{v}_{i}}{k}\right] \tilde{r}^{2}, \\
\frac{5}{2} \frac{\partial}{\partial \tilde{r}}\left\{\tilde{r}^{2} \tilde{p}_{e} \frac{\partial}{\partial \tilde{r}}\left(\frac{\tilde{p}_{e}}{\tilde{n}_{e}}\right)\right\}=-\frac{m_{e}}{M}\left(\tilde{p}_{e}-\tilde{p}_{i}\right) \frac{\tilde{v}_{e}}{n}\left[\frac{\tilde{v}_{e}}{n}+\frac{\tilde{v}_{i}}{k}\right] \tilde{r}^{2},
\end{gathered}
$$

Here $k, n$ are quantum numbers for nucleus and electronic shell correspondingly; $v_{i}, v_{e}$ - frequencies for nucleus and electronic transitions correspondingly.

The following Maple notations on figures are used: v - potential $\tilde{\psi}, \mathrm{t}$ - independent variable $\tilde{r}$, $\tilde{\psi} \leftrightarrow v, \tilde{n}_{e} \leftrightarrow s(t), \quad \tilde{n}_{i} \leftrightarrow r(t), \quad \tilde{p}_{i}(\tilde{r}) \leftrightarrow p(t), \tilde{p}_{e}(\tilde{r}) \leftrightarrow q(t), \quad \tilde{m}_{i} \leftrightarrow M, \tilde{m}_{e} \leftrightarrow L$. Explanations placed under all following figures. The system of external parameters $\tilde{m}_{e}=\frac{m_{e}}{M}, \tilde{m}_{i}=\frac{m_{i}}{M}, A=\frac{\psi_{0}^{2}}{4 \pi r_{0}^{2} p_{0}}, \tilde{v}_{i}, \tilde{v}_{e}$, $k, n$, leads to the vast possibilities of mathematical modeling. Some typical calculations are displayed on figures $5.15-5.24$.

In all cases the following Cauchy conditions were used: $\tilde{\psi}(0) \leftrightarrow v(0)=1, \quad \tilde{n}_{i}(0) \leftrightarrow r(0)=1$, $\tilde{n}_{e}(0) \leftrightarrow s(0)=0, D\left(\tilde{p}_{i}\right)(0)=0, D\left(\tilde{p}_{e}\right)(0)=0, D\left(\tilde{n}_{i}\right)(0)=0, D\left(\tilde{n}_{e}\right)(0)=0, D(\tilde{\psi})(0)=0 . \quad$ Other explanations placed under all following figures. 


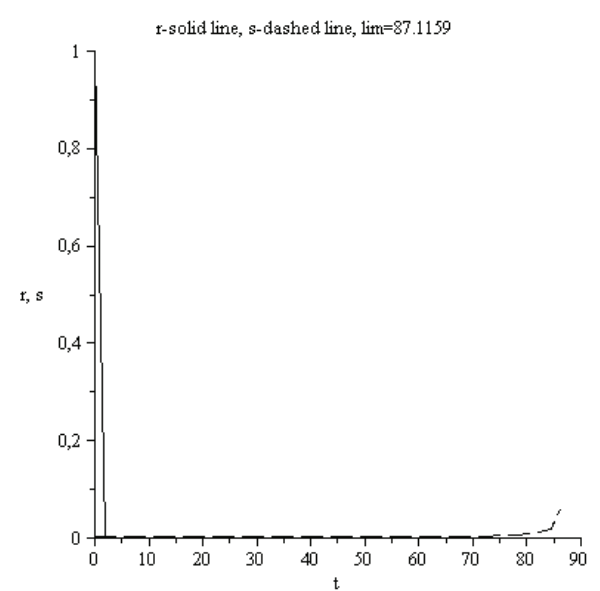

Figure 5.15. Dependence $\mathrm{r}(\mathrm{t}) \leftrightarrow \tilde{n}_{i}(\tilde{r})$ - solid line, $\mathrm{s}(\mathrm{t}) \leftrightarrow \tilde{n}_{e}(\tilde{r})$ - dashed line, $\tilde{p}_{i}(0)=0.01, \tilde{p}_{e}(0)=1$, $\tilde{r}_{\lim }=87.1159 . k=n=1$

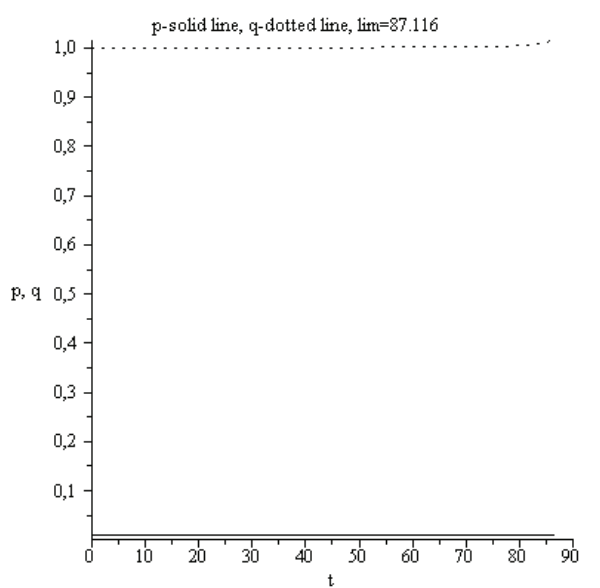

Figure 5.16. Dependence $\mathrm{p}(\mathrm{t}) \leftrightarrow \tilde{p}_{i}(\tilde{r})$ - solid line, $\mathrm{q}(\mathrm{t}) \leftrightarrow \tilde{p}_{e}(\tilde{r})$ - dotted line, $\tilde{p}_{i}(0)=0.01, \tilde{p}_{e}(0)=1$, $\tilde{r}_{\lim }=87.116 . k=n=1$

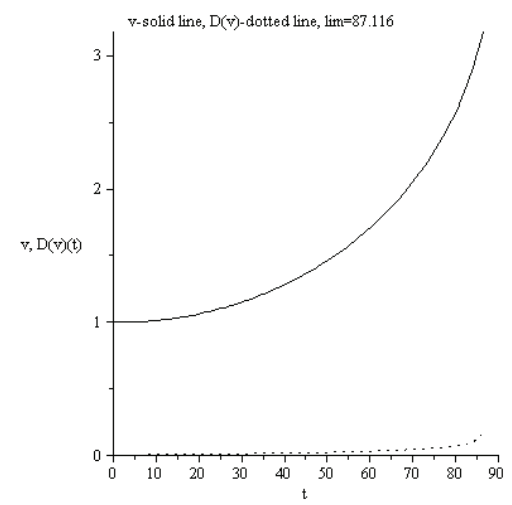

Figure 5.17. Dependence $\mathrm{v}(\mathrm{t}) \leftrightarrow \tilde{\psi}_{i}(\tilde{r})$ - solid line, $\mathrm{D}(\mathrm{v})(\mathrm{t}) \leftrightarrow \partial \tilde{\psi}(\tilde{r}) / \partial \tilde{r}$ - dotted line, $\tilde{p}_{i}(0)=0.01$, $\tilde{p}_{e}(0)=1, \tilde{r}_{\lim }=87.116 . k=n=1$ 


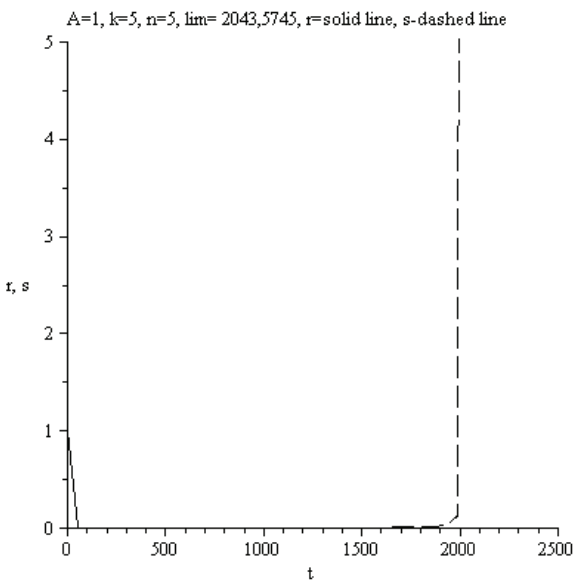

Figure 5.18. Dependence $\mathrm{r}(\mathrm{t}) \leftrightarrow \tilde{n}_{i}(\tilde{r})$ - solid line, $\mathrm{s}(\mathrm{t}) \leftrightarrow \tilde{n}_{e}(\tilde{r})$ - dashed line, $\tilde{p}_{i}(0)=0.01, \tilde{p}_{e}(0)=10$, $\tilde{r}_{\lim }=2043.5745 . k=n=5$

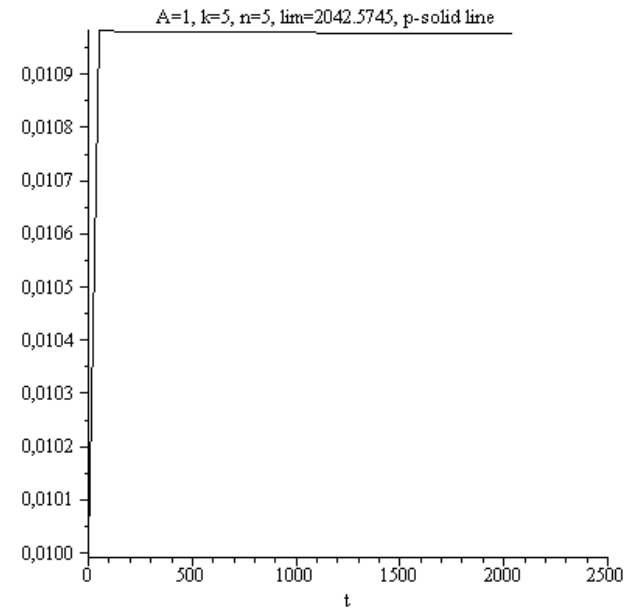

Figure 5.19. Dependence $\mathrm{p}(\mathrm{t}) \leftrightarrow \tilde{p}_{i}(\tilde{r})$ - solid line, $\tilde{p}_{i}(0)=0.01, \tilde{p}_{e}(0)=10, \tilde{r}_{\lim }=2043.5745, k=n=5$

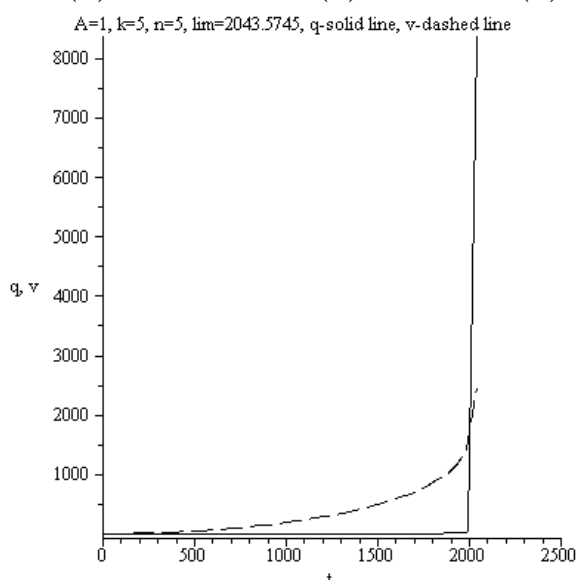

Figure 5.20. Dependence $\mathrm{v}(\mathrm{t}) \leftrightarrow \tilde{\psi}_{i}(\tilde{r})$ - dashed line, $\mathrm{q}(\mathrm{t}) \leftrightarrow \tilde{p}_{e}(\tilde{r})$ - solid line, $\tilde{p}_{i}(0)=0.01, \tilde{p}_{e}(0)=10$, $\tilde{r}_{\lim }=2043.5745 . k=n=5$. 


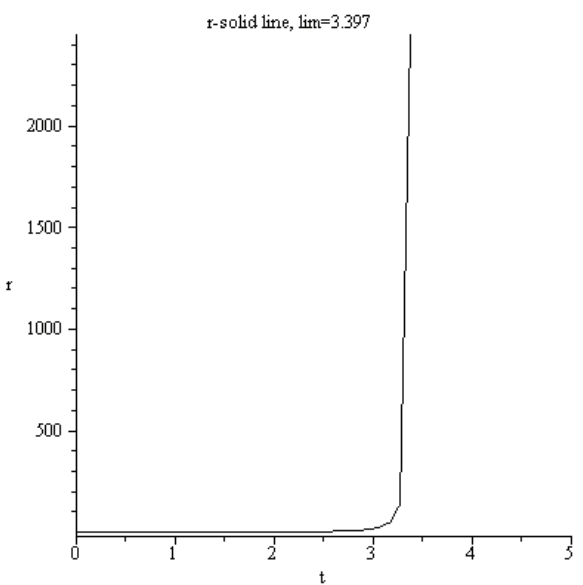

Figure 5.21. Dependence $\mathrm{r}(\mathrm{t}) \leftrightarrow \tilde{n}_{i}(\tilde{r})$ - solid line, $\tilde{p}_{i}(0)=2, \tilde{p}_{e}(0)=0.01, \tilde{r}_{\lim }=3.397$.

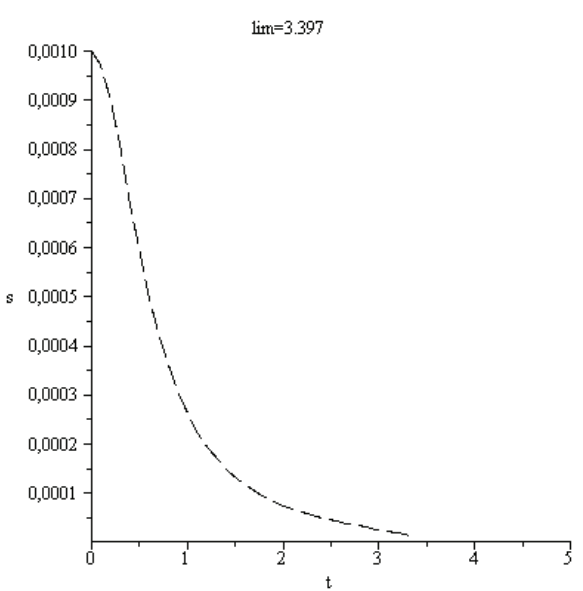

Figure 5.22. Dependence $\mathrm{s}(\mathrm{t}) \leftrightarrow \tilde{n}_{e}(\tilde{r})$ - dashed line, $\tilde{p}_{i}(0)=2, \tilde{p}_{e}(0)=0.01, \tilde{r}_{\lim }=3.397$.

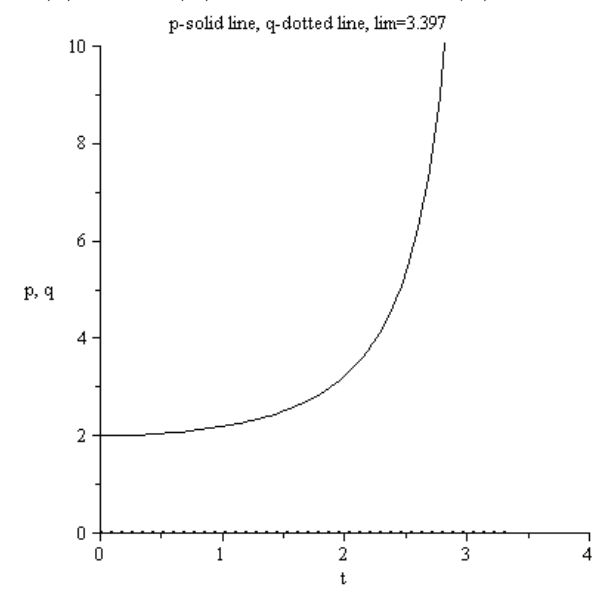

Figure 5.23. Dependence $\mathrm{p}(\mathrm{t}) \leftrightarrow \tilde{p}_{i}(\tilde{r})$ - solid line, $\mathrm{q}(\mathrm{t}) \leftrightarrow \tilde{p}_{e}(\tilde{r})$ - dotted line, $\tilde{p}_{i}(0)=2, \tilde{p}_{e}(0)=0.01$ $\tilde{p}_{e}(0)=0.01, \tilde{r}_{\lim }=3.397$. 


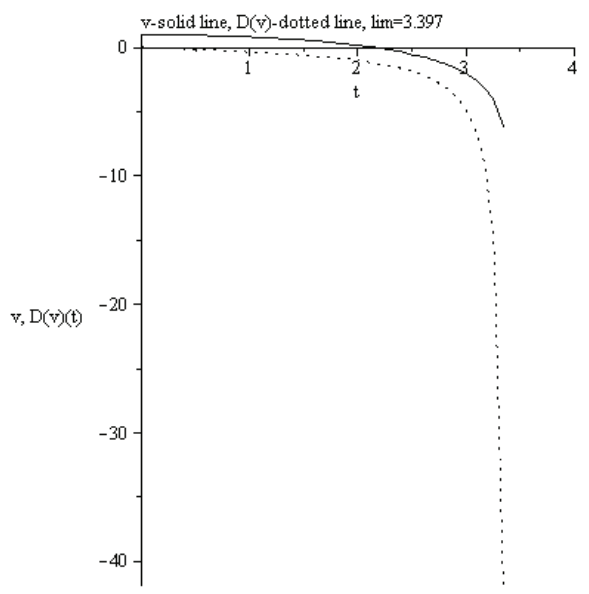

Figure 5.24. Dependence $\mathrm{v}(\mathrm{t}) \leftrightarrow \tilde{\psi}_{i}(\tilde{r})$ - solid line, $\mathrm{D}(\mathrm{v})(\mathrm{t}) \leftrightarrow \partial \tilde{\psi}(\tilde{r}) / \partial \tilde{r}$ - dotted line, $\tilde{p}_{i}(0)=2$, $\tilde{p}_{e}(0)=0.01, \tilde{r}_{\lim }=3.397$.

As it follows from the calculations, the separated charges in plasmoid can correspond to the model of the spherical capacitor. The maximum energy that can be stored in a capacitor is limited by the breakdown voltage. But the breakdown process can have rather lengthy character realized in the several stages. This fact can explain the anomalies in the Tunguska Object (TO) behavior. TO can be considered as a giant atmospheric plasmoid - BL.

The spherical capacitor energy $W$ is written as

$$
W=2 \pi \varepsilon_{0} \varepsilon \frac{R_{1} R_{2}}{R_{2}-R_{1}}(\Delta \psi)^{2}
$$

where $\Delta \psi=\psi_{1}-\psi_{2}$, is the potential difference between the conductors for a given charge $q$ on each. The voltage between the spheres can be found by integrating the electric field along a radial line:

$$
\Delta \psi=\psi_{1}-\psi_{2}=\frac{q}{4 \pi \varepsilon_{0} \varepsilon} \int_{R_{1}}^{R_{2}} \frac{d r}{r^{2}}=\frac{q}{4 \pi \varepsilon_{0} \varepsilon}\left(\frac{1}{R_{1}}-\frac{1}{R_{2}}\right),
$$

If $R_{2}>R_{1}$, then

$$
W=2 \pi \varepsilon_{0} \varepsilon R_{1}(\Delta \psi)^{2}
$$

The force $\mathbf{F}=-\frac{d W_{p}}{d r} \frac{\mathbf{r}}{r}$, acting on the internal conductor

$$
F_{\text {in }}=-\frac{\partial W_{p}}{\partial R_{1}} \cong 2 \pi \varepsilon_{0} \varepsilon(\Delta \psi)^{2},
$$

does not depend on the radius of the internal sphere in the first approximation. For the external sphere, the force acts in the opposite direction.

$$
F_{\text {ex }}=-\frac{\partial W_{p}}{\partial R_{2}}=-2 \pi \varepsilon_{0} \varepsilon_{1}(\Delta \psi)^{2} \frac{R_{1}^{2}}{\left(R_{2}-R_{1}\right)^{2}} .
$$

If $R_{2}>R_{1}$, then

$$
F_{\text {ex }}=-\frac{\partial W_{p}}{\partial R_{2}}=-2 \pi \varepsilon_{0} \varepsilon_{1}(\Delta \psi)^{2}\left(\frac{R_{1}}{R_{2}}\right)^{2},
$$

If for the TO $W=10^{16} \mathrm{~J}$, radius of the internal sphere is $100 \mathrm{~m}, \varepsilon=1$, then $\Delta \psi=1.34 \cdot 10^{12} \mathrm{~V}$.

Electrostatic generator (which uses a moving belt to accumulate very high amounts of electrical potential on a hollow metal globe on the top of the stand) was invented by American physicist Robert J. 
Van de Graaf in 1929. The potential difference achieved in Van de Graaff generators reaches $7 \cdot 10^{6}$ volts in the 30th of the last century.

A Marx generator (Arkadyev - Marks generator in the Russian scientific literature) generates a highvoltage pulse. The circuit generates a high-voltage pulse by charging a number of capacitors in parallel, then suddenly connecting them in series. Marx generators are used in high energy physics experiments, as well as to simulate the effects of lightning on power line gear and aviation equipment. The highvoltage pulse can reach up to $10^{7} \mathrm{~V}$. The mega-joule estimates are known for the ball lightnings.

\section{Conclusion}

We applied the nonlocal physical description for creation of the theory of BL - plasmoid. Nonlocal physics admits the BL existence of two kinds: a) as polarized plasma objects with the positive or negative shell (see figures $5.1-5.8$ ), b) as a macroscopic atom structure with the separated nucleus and shell. In particular we demonstrate the solution of non-local equations for the spherically symmetric plasma object; this object is in the rest, the external magnetic field is absent, but the self- consistent electric field is taken into account.

The solution has the self-consistent character and no needs to formulate the boundary conditions on infinity. BL can exist as a polarized plasma object. The solitons have the character of quantum objects if the initial pressures (Cauchy conditions) of non-local origin for the positive and negative components are not equal each other and objects reach stability as result of equalizing of corresponding pressure of the non-local origin and the self-consistent electric forces. If the initial pressures of non-local origin for the positive and negative components are equal each other, objects lose stability (figures 5.9, 5.10)

The mentioned solutions lead to appearance of BL (atom) structure like solitons with the separated negative and positive parts placed in the finite space domain.

In asymptotic even no needs to use nonlocal parameters. In the second approximation when the approximation for the collision energy integral is introduced we obtain the effective method for the selfconsistent calculation of the atom (BL) structure with the simultaneous quantization for nucleus and electronic shell. For example, the domain size $\tilde{r}_{e l}<\tilde{r}_{\lim }$ is a result of the self-consistent solution. Really, the absolute electron charge $q_{e l}$ is equal to $\left|q_{e l}\right|=e \int_{0}^{r_{e l}} 4 \pi r^{2} n_{e}(r) d r$. This relation defines the atom radius $r_{e l}$. The most significant effect is exerted by the difference pressure values $\tilde{p}_{i}(0),, \tilde{p}_{e}(0)$. An increase in pressure $\tilde{p}_{i}(0)$ (excitation of the nucleus in quantum model) leads to its explosion. These effects can be considered also as explanation of the existence of atom structures.

The time of the numerical calculations is very small. Of course the GHE can be applied also for numerical calculations of many dimensional non-stationary structures.

The theory and its consequences allow us to explain all the properties of ball lightning formulated at the beginning of paragraph 5 .

The delivered theory demonstrates the great possibilities of the generalized quantum hydrodynamics in investigation of the quantum solitons. The usual Schrödinger' quantum mechanics cannot be useful in this situation, because Schrödinger - Madelung quantum theory does not contain the energy equation in principal.

Let us note once again as a final conclusion that the theory of plasmoids (ball lightnings) cannot be constructed within the framework of local physics, and the theory itself is a deep special case of nonlocal physics.

\section{References}

1. Alexeev B.V., Generalized Boltzmann Physical Kinetics. Elsevier Amsterdam, The Netherlands (2004) 368p.

2. Alexeev B.V., Generalized Quantum Hydrodynamics and Principles of Non-Local Physics, J.Nanoelectron. Optoelectron. 3,143 (2007).

3. Alexeev B.V., Unified Non-local Theory of Transport Processes, Elsevier Amsterdam, The Netherlands (2015) $644 \mathrm{p}$. 
4. Alexeev B.V., Unified Non-local Relativistic Theory of Transport Processes, Elsevier Amsterdam, The Netherlands (2016) 455p.

5. Alexeev B.V., Nonlocal Astrophysics. Dark matter, Dark Energy, Physical Vacuum. Elsevier Amsterdam, The Netherlands (2017) 454p.

6. Madelung E. Eine anschauliche Deutung der Gleichung von Schrödinger // Naturwissenschaften. 1926. Bd. 14. S. 1004-1004.

7. Bohm D. Quantum Theory, New York, Prentice-Hall, Inc., 1952.

8. Chapman S., Cowling T.G., "The Mathematical Theory of Non-uniform Gases", Cambridge: At the University Press, (1952).

9. Bell J.S., On the Einstein Podolsky Rosen paradox, Physics, 1, 195 (1964).

10. Gulin A.V., Samarskii A.A. On some results and problems in the theory of stability of difference schemes. Matematicheskii sbornik, v. 99(141), N3, p. 299 - 321, 1976.

11. Boltzmann L. Prinzipe der Mechanik. J.-A. Barth, Leipzig, 1897.

12. Brillouin L. La théorie des quanta et l'atome de Bohr. (Chapter 7), Blanchard, Paris, 1922.

13. Stenhoff M. Ball Lightning. An Unsolved Problem in Atmospheric Physics. New York: Kluwer/Plenum, 1999.

14. K. L. Corum, J. F. Corum. Experiments for Creating of Ball Lightning with the Aid of High-frequency Discharge and Electrochemical Fractal Clusters. Soviet Phys. Uspekhi. 1990. V. 160, N. 4, P. 47 - 58 (in Russuan).

15. Silberg P.A. Ball lightning. In: Problems of Atmospheric and Space Electricity. Proc. 3d International Conference on Atmocpheric and Space Electricity. 1963, Montreux, Zwitzerland. Ed. S. C. Coronititi, Amsterdam, Elsevier, 1965. P. $303-322$.

16. Klimov A.I., Bychkov V.L.. High Energetic Long-lived Non-equilibrium Ball Plasmoids in a Gas Flow and in Atmosphere. Proc. $6^{\text {th }}$ Int. Symp. on Ball Lightning (ISBL'99). 1999, Antwerp, Belgium, P. 212 - 215.

17. Grigor'ev A.I., Grigor'eva I.D., Shiriaeva S.O.. Observations of ball lightning and their analysis. In: Khimia Plasmy. Ed. B. M. Smirnov. Moscow: Energoatomizdat, 1993. P. 218 - 248 (in Russian).

18. Amirov A.Kh., Bychkov V.L., Strijev A.Yu.. Principles of Creating and Processing Data Bank: Meteorologic Applications Illustrated with Regard to Ball Lightning Processing. Journ. of Meteorology. 1995, V.20, N. 197, P. $85-93$.

19. Rayle W.D. Ball Lightning Characteristics. NASA Techn. Note -D -3188, 1966

20. McNally J.R., Jr. Preliminary Report on the Ball Lightning. Oak-Ridge Nat. Lab/ No 3938, May 1966.

21. Egely G.. Analysis of Hungarian Ball Lightning Observations. In: Progress in Ball Lightning Research. Ed. A. G. Keul. Proc. VIZOTUM, P. 22. The Vizotum Project, Salzburg, Austria, 1993.

22. Hubert P. Nouvelle Enquete sur la Foudre en Boule - Analyse et Discussion des Resultats. Rapport $\mathrm{PH} / \mathrm{SC} / 96001$, Commisariat a l'Energy Atomique, Service d'Electronique Physique, Centre d'Etudes Nucleairs de Saclay, France, 1996.

23. Dijkhuis G.C. Statistics and Structure of Ball Lightning. Proc. 3d Intern. Symp. on Ball Lightning (ISBL'92), 1992, Los Angeles, USA.

24. Smirnov, The Observational Properties of Ball Lightning //Successes of Physical Sciences. 1992. Vol. 162, № 8. Pр. 43 - 81. (Смирнов Б.М. Наблюдательные свойства шаровой молнии //Успехи физических наук. 1992. T. 162 , № 8. С. 43 - 81.)

25. Stakhanov, I. P. Physical nature of ball lightning. - Moscow: Atomizdat, 1979.

26. Stakhanov I. P. On the physical nature of ball lightning. - Moscow: Energoatomizdat, 1985.

27. Brand W. Der Kugelblitz. - Hamburg: Henri Grand Verlag, 1923.

28. Roth, J. Reece, Ball lightning: what nature is trying to tell the plasma research community// Fusion Technology 1995; V. 27, P. 255-270.

29. Byturin V., Bocharov A., Klimov A.,et.al., Analysis of Non-Thermal Plasma Aerodynamics Effects, 44 AIAA Aerospace Sciences Meeting \& Exhibit. AIAA 2006-1209, 9-12 January 2006, Reno, NV, 1. 2. th. p.8.

30. Klimov A.I., Int. Journ. Unconventional Electromagnetics and Plasmas (UEP), 2011, V.3, N. 1-2, P. 55.

31. Egorov A. I., Stepanov S. I. Long-living plasmoids-analogues of ball lightning, arising in humid air. //Journal of technical physics. 2002. - Vol. 72, N12. p. 104

32. Oreshko A.G., Proc. XVIII Int. Conf. on Gas Discharge and Their Applications, Greifswald 2010, p.526. 